\title{
Unravelling the effects of disease-associated mutations in TDP-43 protein via molecular dynamics simulation and machine learning
}

\author{
Abhibhav Sharma ${ }^{1}$, Pinki Dey ${ }^{2}$ \\ 1. School of Computer and System Sciences, Jawaharlal Nehru University, New Delhi 110067, India. \\ 2. School of Biotechnology and Biomolecular Sciences, University of New South Wales, Sydney 2033, Australia.
}

\begin{abstract}
Over the last two decades, the pathogenic aggregation of TAR DNA-binding protein 43 (TDP-43) is found to be strongly associated with several fatal neurodegenerative diseases such as amyotrophic lateral sclerosis (ALS) and frontotemporal lobar degeneration (FTD), etc. While the mutations and truncation in TDP-43 protein have been suggested to be responsible for TDP-43 pathogenesis by accelerating the aggregation process, the effects of these mutations on the bio-mechanism of pathologic TDP-43 protein remained poorly understood. Investigating this at the molecular level, we formulized an integrated workflow of molecular dynamic simulation and machine learning models (MD-ML). By performing an extensive structural analysis of three disease-related mutations (i.e. I168A, D169G, and I168A-D169G) in the conserved RNA recognition motifs (RRMs) of TDP-43 and we observed that the I168A-D169G double mutant delineates the highest packing of the protein inner core as compared to the other mutations, which may indicate more stability and higher chances of pathogenesis. Moreover, through our MD-ML workflow, we identified the biological descriptors of TDP-43 which includes the interacting residue pairs and individual protein residues that influence the stability of the protein and could be experimentally evaluated to develop potential therapeutic strategies.
\end{abstract}

Keywords: TDP-43 protein, RRM1 motif, mutations, molecular simulations, machine learning

\section{Introduction}

The molecular mechanisms leading to the accumulation of TAR DNA-binding protein 43 (TDP-43) in the central nervous system is a key feature of several common neurological disorders in ageing societies, such as frontotemporal dementia (FTD), Alzheimer's disease (AD), amyotrophic lateral sclerosis (ALS) and limbic predominant age-related TDP-43 encephalopathy (LATE) ${ }^{1}$. The TDP-43 protein is highly conserved and plays a significant role in RNA regulation such as splicing, transcriptional regulation, mRNA stabilization $^{2,3}$, etc. Moreover, the TDP-43 is a ubiquitously expressed member of the large heterogeneous nuclear ribonucleoprotein (hnRNP) family that shows specific RNA/DNA binding ability by the highly conserved RNA recognition motifs (RRMs) of the proteins ${ }^{4}$. But during pathological conditions, several 
post-translational modifications occur in the protein that leads to their cytoplasmic aggregation causing TDP-43 proteinopathies ${ }^{5-7}$. In fact, $\sim 97 \%$ of all the cases of ALS, $\sim 75 \%$ of patients with severe AD and $\sim 45 \%$ of all the cases of FTDL involve the aggregation of TDP- $-43^{8,9}$. And all the four diseases which are together known as TDP-proteinopathies ${ }^{2,10}$ constitute the major cause of dementia in the world and are expected to rise notoriously in the coming years ${ }^{11}$.

Over the past years, numerous studies were performed to understand the pathological mechanisms underlying TDP-43, and most of the studies focused on the misfolding ${ }^{8}$, mislocalization $^{12}$ and aggregation ${ }^{13-}$ ${ }^{15}$ of the TDP-43 protein. Several studies were able to depict certain mutations in the C-terminal regions of TDP-43 such as R361S, N345K, Q343R that lead to its toxic aggregation in the cytoplasm ${ }^{1,8,16-18}$. Certain mutations in TDP-43 such as A382T, A315T, M337V were also reported to escalate its cytoplasmic mislocalization ${ }^{17,}{ }^{19}$. Most of these mutations are introduced as post-translational modifications, the most common being phosphorylation and ubiquitination of the protein ${ }^{20}$. However, the underlying mechanisms leading to the aggregation of TDP-43 remain elusive. One of the prominent hallmarks of TDP-43 induced proteinopathies is marked by its depletion in the nucleus and increased aggregation into cytoplasmic inclusion bodies. And the rate-limiting step of this process is the cleavage of TDP-43 and generation of Cterminal fragments by the cysteine proteases, caspase and calpain ${ }^{21,22}$.

The generation of C-terminal fragments, TDP-25 (25kD fragment) and TDP-35 (35kD fragment) by caspase- 3 and caspase- 7 is the most prominent step for the clearance of TDP- $43^{23}$. Several experimental studies have also reported a significant delay in cell death on blockage of the caspase digestion ${ }^{23-25}$. Moreover, it is shown that out of the four prominent caspase cleavage consensus sites, three sites lie in the RNA recognition motifs (RRM) of the TDP-43 protein $^{26}$. The cleavage at D89-A90 of N-terminal domain (NTD) of TDP-43 generates TDP-35 that is still capable of folding correctly ${ }^{27}$. But, both the cleavage sites at D169-G170 and D174-C175 of the RRM of TDP-43 generate TDP-25 which lacks the NTD, nuclear localization signal (NLS) and most of RRM1, trapping the protein in the cytoplasm ${ }^{28}$ and thus enhancing its cytoplasmic aggregation ${ }^{29}$. Interestingly, certain mutations, particularly D169G in the RRM1 domain is reported to increase the thermal stability of the protein which becomes more accessible to cleavage by caspase-3 resulting in the early onset of diseases such as ALS ${ }^{26}$. They also showed that the neighbouring I168 residue is also very crucial for protein folding ${ }^{26}$. Recently, mutations in the RRMs are also shown to influence the DNA or RNA binding specificities ${ }^{30}$ indicating the role of nucleic acid-binding in TDP-43 aggregation. However, studies on the effect of disease-related mutations on the RRM domains of the TDP43 is still very limited. Given the crucial role played by the disease-causing mutations in the RRM domain in regulating the protein conformational stability, nucleic acid binding and their role in affecting the cleavage sites remains largely unexplored. 
In this paper, we perform extensive structural analysis on the impact of disease-related mutations in the RRM1 domain of the TDP-43 protein. We address this question by formulizing a framework MD-ML, that integrate molecular dynamics and machine learning approaches in mainly two ways (i) structural analysis of the wild type and disease-causing mutant proteins bound to the nucleic acid by molecular dynamics simulations and (ii) identifying the functionally important regions and biological descriptors of TDP-43 in different mutated states that are crucial in explaining the effect of disease-causing mutations in the caspase cleavage sites in RRM motifs of TDP-43 using Machine Learning models. In this direction, the in-silico approach of molecular dynamics simulation holds promising aspects as it provides insights at the molecular level by mimicking the physicochemical changes that occur within the biomolecule when subjected to a specific condition. However, the high dimensional nature of biomolecular simulation data makes it challenging to extract the discriminatory features or set of collective variables (CVs) of the system ${ }^{31}$. In our case, the CVs that we are interested in are those optimum sets of descriptors that discriminate the different biomolecular states (wild type and mutated) over a specific time scale. Recently, machine learning methods are receiving great attention in the biological research domains including sequence structure-function prediction, genomics, and biological imaging ${ }^{32-34}$. Machine learning models have shown esoteric capabilities of learning the ensemble properties through the MD simulation data and subsequently were able to predict the biomolecular functions ${ }^{35}$ as well as the optimum set of CVs or important discriminating molecular descriptors such as long-distance interaction ${ }^{36}$ that govern the biomolecular changes during the trajectory.

\section{Material and Methods}

\subsection{Molecular Dynamic Simulation}

To investigate the structural changes induced by the disease-causing mutants on the nucleic acid bound RRM1 domain of TDP-43 proteins, we performed 100ns long molecular dynamics (MD) simulations of the wild-type and three TDP-43 mutant proteins with D169G, I168A-D169G, I168A mutations in their RRM1 domain. We selected these mutations as they are experimentally found to impact the overall stability of the protein $^{26}$. We adopted the crystal structure of human TDP-43 in complex with a single-stranded DNA (PDB ID: 4Y0F.pdb) for the wild-type protein and crystal structure of human TDP-43 (PDB ID: 4Y00.pdb) for the D169G mutant protein ${ }^{26}$. Due to the unavailability of crystal structures, the I168A-D169G, I168A mutations were introduced into the wild-type protein using the PyMol's Mutagenesis Wizard. The Isoleucine and Aspartic acid at positions 168 and 169 in the wild type were replaced by Alanine and Glycine respectively in I168A-D169G TDP-43 mutant whereas the I168A TDP-43 mutant is obtained by replacing Isoleucine at position 168 to Alanine. The RRM1 protein used in our study is composed of 78 amino acids and 10 nucleotides. A schematic representation of the protein structures and the position of mutations with 
respect to the cleavage site is shown in Fig. 1A. All the MD simulations were carried out using the GROMACS software (version 2019.3) and AMBER03 protein, nucleic AMBER94 ${ }^{37}$ forcefield. Each wild type and mutant system were solvated using a simple point charge (SPC) water model and were neutralized by adding $\mathrm{Na}+$ and $\mathrm{Cl}$ - ions. Each solvated system was subjected to 50,000 steps of energy minimization and 100ps each of NVT and NPT equilibrations. The temperature was kept constant using a Berendsen thermostat and the pressure was fixed at 1 bar using The Parrinello-Rahman algorithm ${ }^{38}$. Further, 100ns long MD simulations were carried out at a mean temperature of $300 \mathrm{~K}$ and $\mathrm{pH} 7$ with an integration time step of $2 \mathrm{fs}$. The simulations were carried out under periodic boundary conditions and particle mesh Ewald treated the long-range electrostatic interactions.

The analyses were performed using the GROMACS utilities and the coordinate dataset generated through the MD simulation was used to generate (i) the average contact-map for the entire trajectory of each state (wild type and mutants) where the contact between every $i^{\text {th }}$ and $i+4^{\text {th }}$ residues were calculated with a cutoff distance of $10 \AA$. (ii) difference of the average pairwise distances of the residues in the mutant proteins with respect to the WT protein and (iii) Dynamic cross-correlation (DCC) analysis was carried out to intuitively unravel the functionally relevant regions of protein states throughout the trajectory. Within the given molecule the DCC metric for a pair of residues represents the degree to which these residues move together and is calculated as ${ }^{39}$ :

$\operatorname{DCC}(i, j)=\frac{\left\langle\Delta r_{i}(t) \cdot \Delta r_{j}(t)\right\rangle_{t}}{\sqrt{\left\langle\left\|\Delta r_{i}(t)\right\|^{2}\right\rangle \cdot\left\langle\left\|\Delta r_{j}(t)\right\|^{2}\right\rangle}}$

where $\mathrm{r}_{\mathrm{i}}(\mathrm{t})$ is the vector of the $\mathrm{i}^{\text {th }}$ atom's coordinates as a function of time $t,\langle *\rangle$ denotes the time ensemble average and $\Delta r_{i}(t)=r_{i}(t)-\left\langle r_{i}(t)\right\rangle_{t}$. Although the contact-map and DCC approach highlighted some ambiguous yet important fingerprints of each state, these approaches however provided little information to unmask the intricate intra- and inter-molecular relations and features that are critical for understanding the effect of different types of mutations in the protein.

\subsection{The Machine Learning Utilization}

The dataset generated by the MD simulation is high dimensional, extraneously noisy and encompasses a set of important features and patterns encrypted within that could explain the changes that occur within the biomolecular ensemble. Machine learning models carry promising potentials to identify these discriminatory features and fingerprints that could elevate our understanding of the key mechanisms underlying the behaviour of biomolecules in the specific state. 


\subsubsection{Data Pre-processing}

There are several ways in which the MD simulation dataset can be utilized to extract features. For a large system, the internal coordinates of residues could be used as feature variables but often tend to produce fallible conclusions due to overfitting ${ }^{36}$. For a small or moderate size ensemble, such as the TDP-43 DNA complex in our case, the distance-based features would provide much deeper insights as it contains a profuse amount of conformational and physicochemical information of the system ${ }^{36}$. Here we incorporated the inverse inter-atomic distance as features because it also highlights the significant local changes within the ensemble. For amino acid, the $\mathrm{C}_{\mathrm{a}}$ and for nucleotides, the centre of mass was used to measure the inverse inter-residue distance. The total of 88 residues including that of the RRM1 domain and the nucleotides resulted in 88(88-1)/2 variables. The 2000 frames generated for each type of state thus yield an inversedistance dataset of size $2000 \times 3828$. To meticulously probe the effects of the mutations, we adopted two approaches (Fig. 1E) i.e. (i) To study the individual effects of the point mutations on TDP43, we performed the feature extraction by pairwise concatenation of the datasets of each type of mutated state (i.e. D168G, D168G-I169A and I169A) with the wildtype (WT) dataset ; (ii) To unmask the globally important interactions and discriminatory $\mathrm{CVs}$, we concatenated the labelled inverse-distance dataset of all the mutated and wild type states. To subdue the curse of dimensionality, we included only those interaction pairs as variables whose interatomic distance was greater than $10 \AA$ but also have a distance less than $10 \AA$ in at least one frame.

\subsubsection{Machine Learning models}

Here, we employed supervised machine learning models over the simulation datasets - (Objective I) to extract the highly discriminatory features (i.e., interacting pairs) that distinguish the mutated states from the wildtype state of TDP43 and its binding; (Objective II) to depict the importance per residue profile of TDP-43 that unmasked the biological descriptors at the residue level. For the former, we employ two feature extraction models L1 regularization regression model (LASSO) and Extreme Gradient Boosting Machine (XGBoost). For the latter, we incorporate L1- L2 regularization regression model (Elastic Net) and Random Forest (RF) models (Fig. 1E). Based on their scaled scoring scheme, these models would extract important pairs of interactions but the relevance per residue was computed by averaging the importance score of all the pairs involving that residue followed by normalization between 0 and 1 . Models were trained and tested over the concatenated dataset where each frame was labelled to its corresponding state. For feature selection, we only corroborate the supervised learning model because, unlike unsupervised models, supervised learning models are known for their esoteric capabilities of exploiting the class information to learn the encrypted discriminatory features and subtle patterns within the dataset that highlights the key differences between given classes. 


\subsubsection{Regularized Regression Models}

Least Absolute Shrinkage and Selection Operator (LASSO) is a regularized regression model from the family of generalized linear models. By penalizing the (L1-norm) regression model, LASSO reduces the regression coefficient to zero for those features that have low contribution in the learning of the model. This makes LASSO a pervasive feature selection model. Alternative to LASSO regression, Ridge regression is also based on the model penalization, but it involves an L2-norm ${ }^{40}$.

For a given feature dataset $\mathrm{D}$, let $x_{i j}$ be the observation of the $j^{\text {th }}$ variable $(1<j \leq p)$ in the $i^{\text {th }}(1<i \leq$ $N$ ) frame and consider $y_{i}$ be the corresponding state label of the $i^{\text {th }}$ frame. In addition to minimizing the sum of squared error, the regularized model learns the regression coefficient $\beta_{j}$ for each $j^{\text {th }}$ variable including the intercept $\beta_{0}$ (eq.2) by imposing a constraint on the coefficient $\sum J\left(\beta_{k}\right) \leq t^{41}$.

$\hat{\beta}=\operatorname{argmin}_{\beta} \sum_{i=1}^{N}\left(y_{i}-\beta_{0}-\sum_{j=1}^{p} x_{i j} \beta_{j}\right)^{2}$

LASSO truncates the coefficient of the low-importance to zero features by imposing L1 constraint $J\left(\beta_{k}\right)=$ $\left|\beta_{k}\right|$ ) (eq. 3) while the Ridge shrinks the coefficient close to zero for the low contributing variable by imposing L2 constrain $\left(J\left(\beta_{k}\right)=\beta_{k}^{2}\right)^{42}$.

$\hat{\beta}^{\text {lasso }}=\operatorname{argmin}_{\beta}\left\{\frac{1}{2} \sum_{i=1}^{N}\left(y_{i}-\beta_{0}-\sum_{j=1}^{p} x_{i j} \beta_{j}\right)^{2}+\lambda \sum_{j=1}^{p}\left|\beta_{j}\right|\right\}$

where $\lambda$ is the penalty parameter and we find the best fitting $\lambda$ using cross-validation ${ }^{40}$. While LASSO identifies a set of most discriminating independent inter-residual interaction pairs, it drops all the other noncontributing as well as low-contributing variables and therefore is not suitable to account for per-residue importance for the system. We, therefore, employ an Elastic Net classifier to yield a per-residue importance profile for the mutated systems. Based on penalization, Elastic Net is a smart combination of L1 and L2 constrain with $J\left(\beta_{k}\right)$ (coefficient constrain) given as:

$\lambda \sum_{j=1}^{p}\left(\alpha \beta_{j}^{2}+(1-\alpha)\left|\beta_{j}\right|\right)$

where the $\alpha$ constant governs the intensity of Ridge and LASSO penalties ${ }^{41}$. We used the R package "caret" to train LASSO and Elastic Net models ${ }^{43}$.

\subsubsection{RandomForest}


Breiman et. $\mathrm{al}^{44}$ developed the random forest $(\mathrm{RF})$ algorithm that utilizes the ensemble of decision trees to perform prediction and classification. After bootstrapping the feature dataset with replacement, allowing duplicate entry of the instances, the fully grown classification trees are produced by randomly sampling a set of variables at each split ${ }^{45}$. This way, RF performs feature selection by carrying out a bootstrapping and aggregation (bagging) for tree building. The decisions are then made based on the average of collective decisions by all the fully grown decision trees. For each generated tree, the RF quantifies the performance of that tree by measuring Out-Of-Bag (OOB) error based on the samples that were not included during the bootstrapping. The OOB has a crucial role in estimating the goodness of fit for RF while also exempting us from performing the cross validation. For a decision tree, the Gini impurity metric reflects how good a node is in splitting. In RF, the mean decrease in impurity for an individual variable over all the trees indicates the importance of that feature variable ${ }^{44}$. We used the R package "RandomForest" to train RF model ${ }^{46}$. A detailed description of the RF algorithm is included in the supplementary text.

\subsubsection{XGBoost}

J.H Freidman ${ }^{47}$ introduced gradient boosting algorithms that have shown competitive classification and prediction potentials on many occasions. Based on the gradient boosting algorithm, Chen et al. ${ }^{48}$ proposed a scalable tree boosting machine called extreme gradient boost (XGBoost) that performed remarkably in several biological research ${ }^{49,50}$.

The underlying principle of XGBoost is that for the given data $D=\left\{\left(x_{n}, y_{n}\right)\right\}_{n=1}^{N}$, the $k$ classification trees or stumps given as $F=\left\{f_{1}(x), f_{2}(x) \ldots, f_{k}(x)\right\}$ assigns a class decision score $f_{k}$ to the data instance by passing it through the leaf node as per the division points of the variable. When the data instance $x_{i}$ passes through a leaf node, the $f_{k}$ is assigned and the prediction result is registered. The collective sum of prediction results by each tree accounts for the final prediction result for each instance. The model follows:

$\hat{y}_{i}=\sum_{k=1}^{K} f_{k}\left(x_{i}\right), f_{k} \in F$

Here, $f_{k}\left(x_{i}\right)$ denotes the prediction score of $k^{\text {th }}$ leaf node. The sum of prediction results overall $K$ stumps yields the prediction result $\left(y_{i}\right)$ for the $i^{t h}$ instance $x_{i}$. The Objective function $\operatorname{Obj}(\theta)$ for XGBoost is given as:

$\operatorname{Obj}(\theta)=\sum_{i=1}^{n} l\left(y_{i}, \hat{y}_{i}\right)+\sum_{k=1}^{K} \Omega\left(f_{k}\right)$

Where $\theta$ is the model parameter. The sum $\sum_{i=1}^{n} l\left(y_{i}, \hat{y}_{i}\right)$ accounts for the error that the model accumulates with $l(x, y)$ is the error function. The second summation $\sum_{k=1}^{K} \Omega\left(f_{k}\right)$ represents the model's regularization term, accounting for the complexity of the trees. A thorough overview of the XGboost algorithm is 
described in Wei li et $\mathrm{al}^{51}$. An inclusive pseudocode ${ }^{47}$ for the gradient boosting algorithm is added in the supplementary text. Here we employed XGBoost to identify the most influencing residue interaction pairs for the mutated states. We used an R package "XGBoost" to implement the algorithm ${ }^{52}$.

\subsubsection{Principal Component Analysis (PCA)}

In the paradigm of finding biological descriptors using a simulation dataset, the unsupervised learning models perform worse than the supervised learning model because of their incapability to exploit the state label ${ }^{36}$. While the unsupervised learning models are not suitable for the unmasking of descriptors, they are highly robust in dimension reduction where the feature variables are mapped to a lower-dimensional configurational space and thus highlighting the main characteristics of the trajectory. In this direction, the PCA method is the most celebrated machine learning model that reveals the dominant modes in the motion of biomolecules by incorporating the molecular dynamic simulation trajectory ${ }^{53}$, 54 . These motions reflect the correlated vibrations or collective motion of a set of residues in the trajectory ${ }^{53}$. In principle, For the $\mathrm{N}$ residue system, the 3 -dimensional cartesian coordinate configuration is transformed into a $3 \mathrm{~N} \times 3 \mathrm{~N}$ covariance matrix whose eigenvectors provide vectorial information indicating the direction of each component of the trajectory while the corresponding eigenvalue represents the intensity contribution of that particular component. We elucidated this motion of eigenvectors through "porcupine" plots ${ }^{55}$ for each type of state of TDP43 which depicts the direction and magnitude for each residue in that state. We used PyMol software to generate the porcupine plots ${ }^{56}$.

The computation was performed on an Intel (R) Core (TM) i5-4310 U, 16 GB RAM, and 64-bit OS Win 10 configuration. The $\mathrm{R}$ version 4.0.3. was used to prepare codes for conducting the experiments and is shared in the Github link.

\section{Results and Discussions}

\subsection{Molecular insights into structural changes in protein due to the disease-causing mutations.}

To investigate the structural changes that undergo in the protein due to mutations, we started by analyzing the stability of the single-stranded DNA bound wild-type (WT) and the mutant proteins from the 100ns simulation trajectories. From the RMSD analysis of the proteins (Fig.1C), we see that the overall protein structure is maintained for the wild type and mutant proteins and the mutations in the protein don't impact the overall conformational stability of the protein molecule. However, we see that the D169G mutant protein shows the lowest RMSD as compared to the wild-type protein (Fig.1C). Several experimental studies have indicated that the D169G mutation increases the overall stability of the protein due to enhanced packing or hydrophobic folding of the RRM1 core domain ${ }^{26}$. We also calculated the evolution of solvent accessible surface area (SASA) of the WT and mutant proteins throughout the simulation trajectory and 
bioRxiv preprint doi: https://doi.org/10.1101/2021.07.28.454112; this version posted July 28, 2021. The copyright holder for this preprint (which was not certified by peer review) is the author/funder, who has granted bioRxiv a license to display the preprint in perpetuity. It is made available under aCC-BY-NC-ND 4.0 International license.

found that mutant proteins, especially the I168A-D169G and D169G mutation has a lower SASA indicating stronger hydrophobic interactions of the core residues of RRM1 domain (Fig.1D). 
bioRxiv preprint doi: $h t t p s: / / d o i . o r g / 101101 / 2021.07 .28 .454112$ t this version posted July 28,2021 . The copyright holder for this preprin (which was not certified by peer review) is the author/funder, who has granted bioRxiv a license to display the preprint in perpetuity. It is made available under aCC-BY-NC-ND 4.0 International license.

(A)

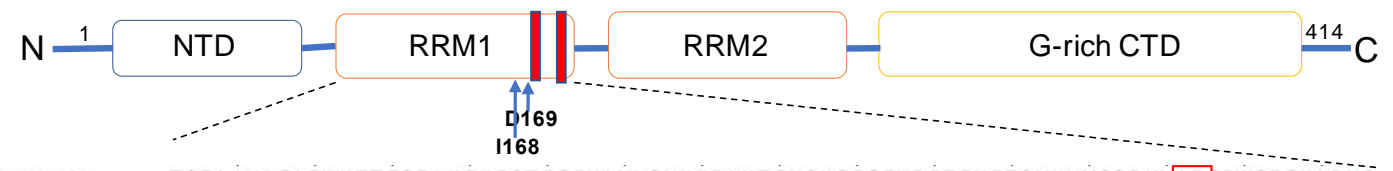

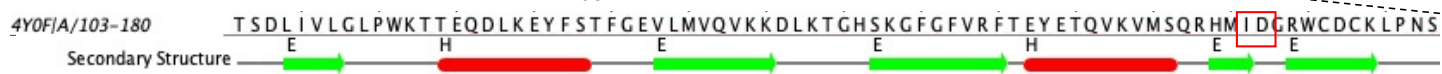

(B)
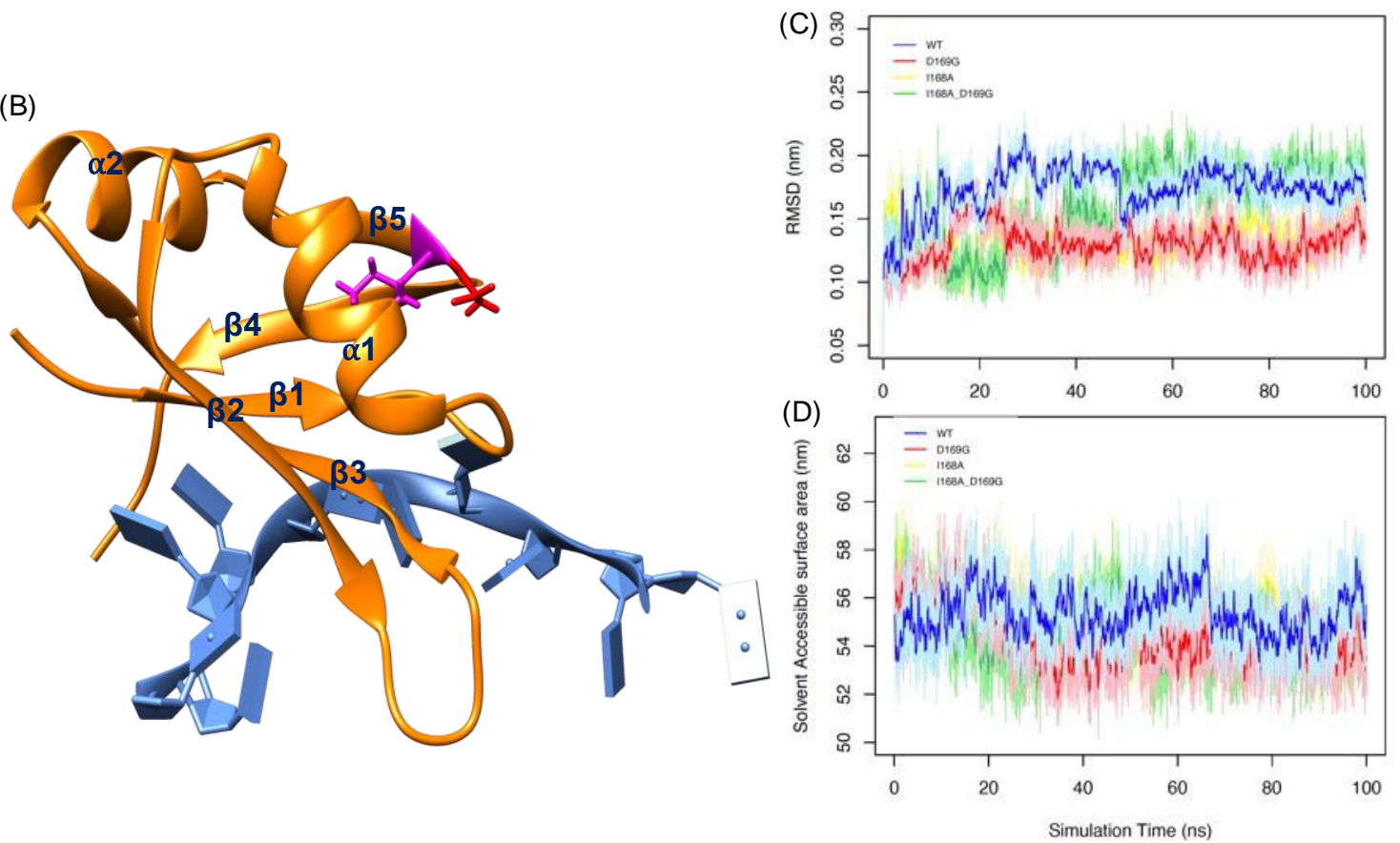

(E)

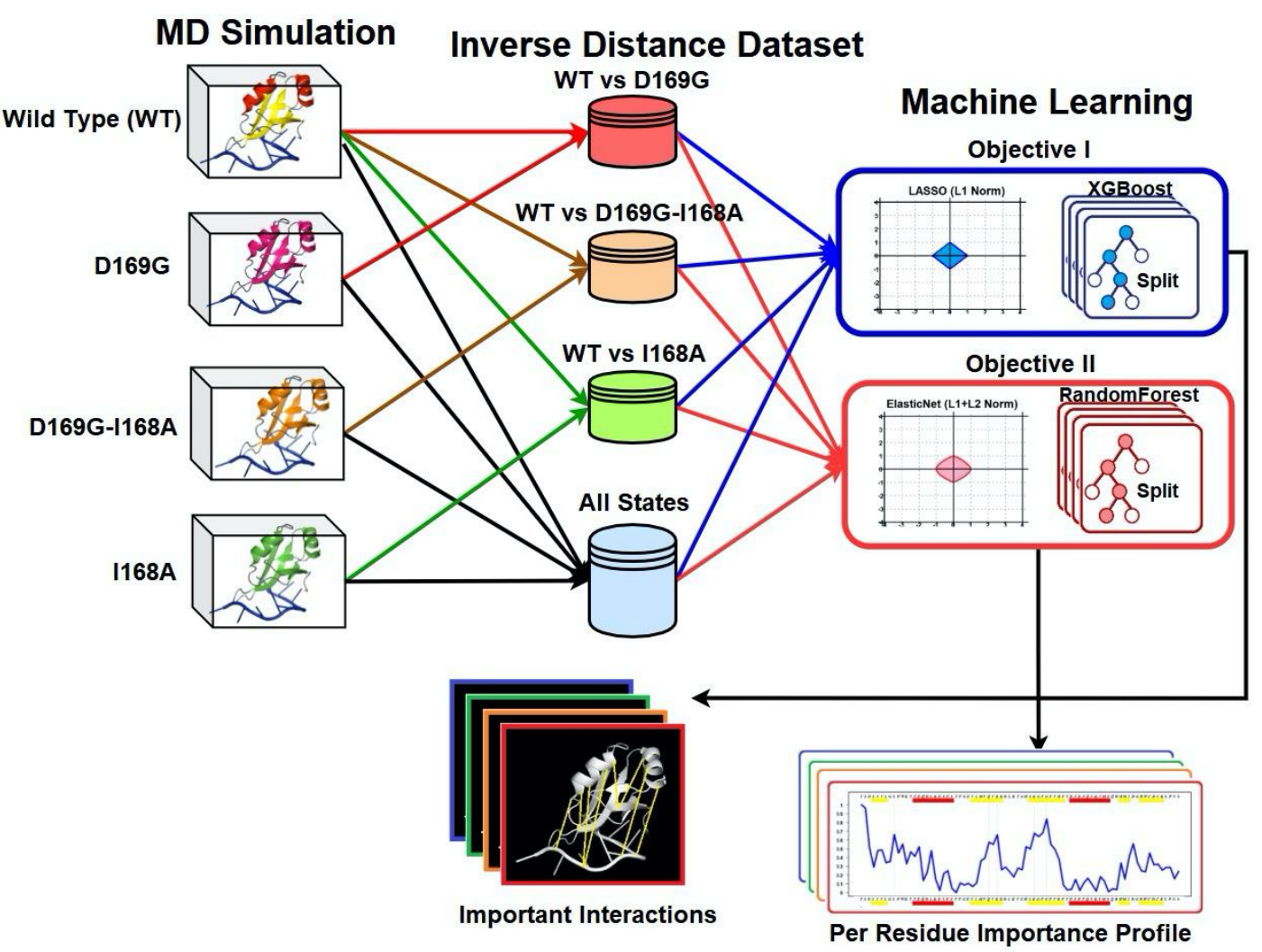

Fig.1: Schematic representation of the DNA bound RRM1 domain of TDP-43 and the MD-ML workflow used for analysing the structural changes in the protein due to mutations. (A) The domain architecture of 
bioRxiv preprint doi: https://doi org/10.1101/2021.07.28.454112; this version posted July 28,2021 . The copyright holder for this preprint (which was not certified by peer review) is the author/funder, who has granted bioRxiv a license to display the preprint in perpetuity. It is made available under aCC-BY-NC-ND 4.0 International license.

TDP-43 consists of an N-terminal domain (NTD) followed by two RNA recognition motifs (RRM1 and RRM2) and a Glycine-rich C-terminal domain. The cleavage sites in the RRM1 domain (D169-G170 and D174-C175) are shown in red strips. The sequence of the RRM1 domain is shown with a red box highlighting the positions of the residues to be mutated. (B) The crystal structure of TDP-43 RRM1 domain (PDB ID: 4Y0F.pdb) in complex with a single-stranded DNA. The RRM1 domain consists of two a stands and five $\beta$ strands. The amino acids that are mutated, i.e., D169G (shown in red), I168A (shown in magenta), I168A-D169G lie in a loop between the $\beta 4$ and $\beta 5$ strand and one of the cleavage sites (D174) are in the $\beta 5$ strand. (C-D) The Root Mean Square Deviation (RMSD) and Solvent Accessible Surface Area (SASA) of the WT and mutant proteins (D169G, I168A, I168A-D169G) are shown as a function of the simulation time(ns). (E) Schematic representation of the MD-ML workflow. The implementation of Machine Learning algorithms over the Molecular Dynamics simulation data to interpret significant biological characteristics.

To further investigate the underlying mutation-induced molecular changes in the protein, we estimated the structural changes occurring in the WT and mutant proteins throughout the simulation time. Firstly, we calculated the difference of the average pairwise distances of the residues in the mutant proteins with respect to the WT protein to view the relative structural changes occurring in the protein due to mutations. The averaging is done over an ensemble of configurations of WT and mutant proteins generated throughout our simulations. From Fig. 2(A, C, E), we see that some of the inter-residue distances increase in the mutant proteins with a simultaneous decrease in other inter-residue distances. Although the global dissemination is observed for all the mutant protein structures, the major impact is seen in the double mutant protein (Fig. $2 \mathrm{C}$ ) where we see that the relative distances of the start (a1 and $\beta 1$ strand) and end regions ( $\beta 4$ and $\beta 5$ strands) of the protein decreases with respect to the mutant protein core ( $\beta 2, \beta 3$ strands). A similar but less profound observation is seen for the D169G mutant protein (Fig. 2A). The relative motion of the mutant proteins with respect to the protein core is shown in the porcupine plots in Fig. 2 (B, D, F). The porcupine plots show the coordinated motion of the $\mathrm{C}_{\alpha}$ atoms in the mutant proteins along their first eigenvectors. The arrowhead shows the direction of motion, and the size of the arrows gives the magnitude of the motion. The porcupine plots further strengthen the observation of the induced movement of the protein terminal regions towards the protein core due to the mutations. After having seen the relative changes in the protein structure due to the mutations, we also analysed if these structural changes in the mutant proteins result in any changes in the contact form within the mutant protein structures throughout the simulation time (Fig. S1AD). To get a more comprehensive outlook from our non-bonded contact formation map, we also included how long a contact is formed between two residues in our analysis. The darker the spot in the contact map, the longer the contact was formed between those pairs of residues. We see that the overall contact formation in the WT and mutant protein structures remains intact but some new contact formations are observed between the a1 and $\beta 5$ strands in the I168A-D169G and I168A mutant proteins. This may be due to the removal of the bulkier isoleucine by alanine that facilitates the protein to attain a more compact structure by promoting contact formation between the a1 and $\beta 5$ strand, mainly between the residues Lys 120 - 
bioRxiv preprint doi: https://doi. org/10.1101/2021.07.28.454112; this version posted July 28, 2021. The copyright holder for this preprint (which was not certified by peer review) is the author/funder, who has granted bioRxiv a license to display the preprint in perpetuity. It is made available under aCC-BY-NC-ND 4.0 International license.

plots. Porcupine plot of (B) D169G (D) I168A- D169G and (F) I168A mutant proteins generated from the first eigenvectors showing the direction of motion of the protein represented by the red arrowheads.

\subsection{Effect of mutations on interactions of the protein with DNA}

Studies have suggested that the disruption of RNA or DNA binding to the RRM1 domain of TDP-43 through mutation or truncation can alter the protein solubility leading to the appearance of aggregates in the nucleus ${ }^{30,58}$. To investigate the effects of mutation on the interaction of the RRM1 domain with the DNA molecule, we calculated the evolution of distance between the WT and mutant proteins with respect to the DNA molecule. Fig. 3 presents the evolution of the average pair-wise distance between the WT and mutant proteins with the DNA throughout the simulation time. We see that the average residual distance between the core (mainly $\beta 4$ and $\beta 5$ ) and the terminal region ( $a 1$ and $\beta 1$ ) of I168A-D169G double mutant protein increases the most as evident from the colour-code representing the higher average residual distance between them (Fig. 3C). We also calculated the protein-DNA contact formation in the WT and mutant protein throughout the simulation time and find that most of the contact form with the DNA takes place between the protein core and initial alpha-helix and beta-strand regions of the protein (Fig. S3A-D). From the propensity of contact formation, we see that the introduction of mutations, especially the I168A leads to the disruption of contacts between the protein core, mainly loop4 (between $\beta 2$ and $\beta 3$ ) and the DNA.

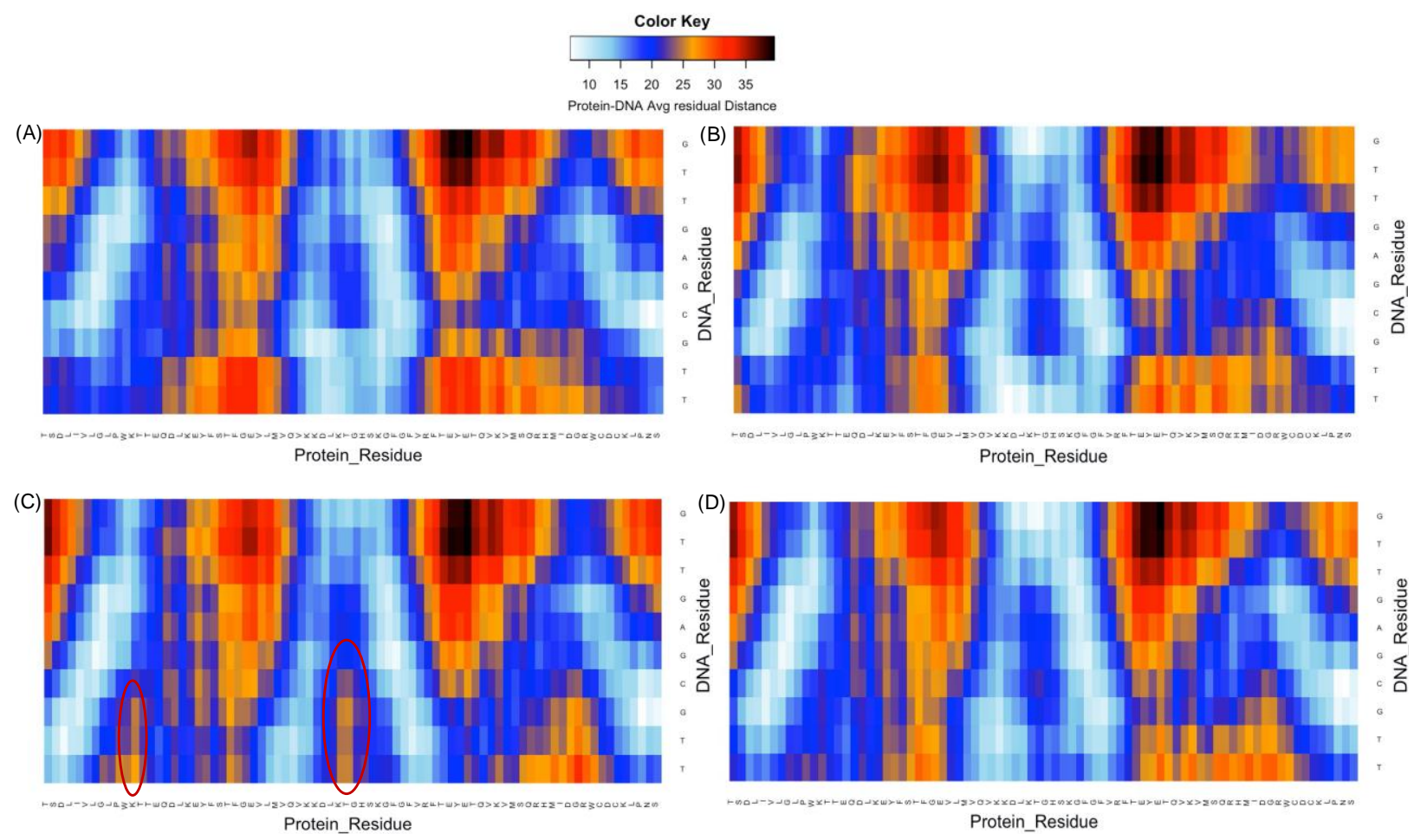

Fig. 3. Average residual distance between the (A) Wild-type (B) D169G mutant (C) I168A-D169G and (D) I168A mutant proteins with the DNA throughout the simulation time. The colour-code represents the 
evolution of the average residual protein-DNA distance from 0 to $50 \AA$. The protein and DNA residues are presented by their single-letter codes.

\subsection{Identifying the biological important descriptors of TDP-43 in different mutated states}

After having seen the structural changes occurring in the protein due to the disease-associated mutations, we now investigate the biological descriptors and the functionally important regions of the protein using Machine Learning models. In this direction, we trained various state-of-the-art supervised learning models to classify the states by learning the ensemble's features. Interestingly, all the supervised learning models have performed excellently in learning the differences among different states of mutation as they attain a high classification accuracy of $99.9 \%$ on a 5 -fold cross-validation test (Table S1). The receiver operating curve (ROC) and area under it (AUC) remained very high as well (Fig. S4), thus empowering the results obtained. The list of the most optimum hyperparameters for each type of learning model that were used is provided in the supplementary (Table $\mathrm{S} 2$ ). While probing all the four types of states together, the regularized regression models (LASSO and ElasticNet) remained time expensive for both objectives. This was also observed when conducting a pair-wise WT vs Mutant state analysis.

\subsubsection{Objective I - Identify the discriminatory features of the RRM1 domain of TDP-43}

To identify the discriminatory features, we employ LASSO and XGBoost on the labelled frame of feature datasets. The importance of each feature is yielded by averaging the importance for the interacting pairs obtained by the models. Figure 4A \& 4C presents the average globally important protein inter-residue and protein-DNA residue interaction map respectively where the importance of interaction is shown by the colour key, where the darker shade represents the higher importance of the interaction. Here, with "globally" we tend to mean that the analysis was carried out by training the features of all the mutant states together. The importance interaction map for the pair-wise analysis of each mutant state is provided in the supplementary (Fig. S5). Some of the important protein-protein and protein-DNA interactions are shown in the cartoon representations (Fig. 4B \& 4D) of the protein complex. For the protein, we find that most of the important discriminating interactions involve the residues in the protein core (Fig. 4A). The residue pairs having the higher important discriminating scores were found to be M132 - V135, L120 - M132, L111-K136, D119-V133. These interactions can be experimentally assessed by biomedical researchers to develop potential therapeutics for the TDP-43 associated diseases. This also indicates the crucial role played by the protein's inner core in maintaining the stability of the protein and its interaction with the DNA. These results obtained through the machine learning models are in line with the studies that have suggested that the D169G mutant enhances the stability of TDP-43 by increasing the hydrophobicity or compactness of the core of the RRM1 domain. 

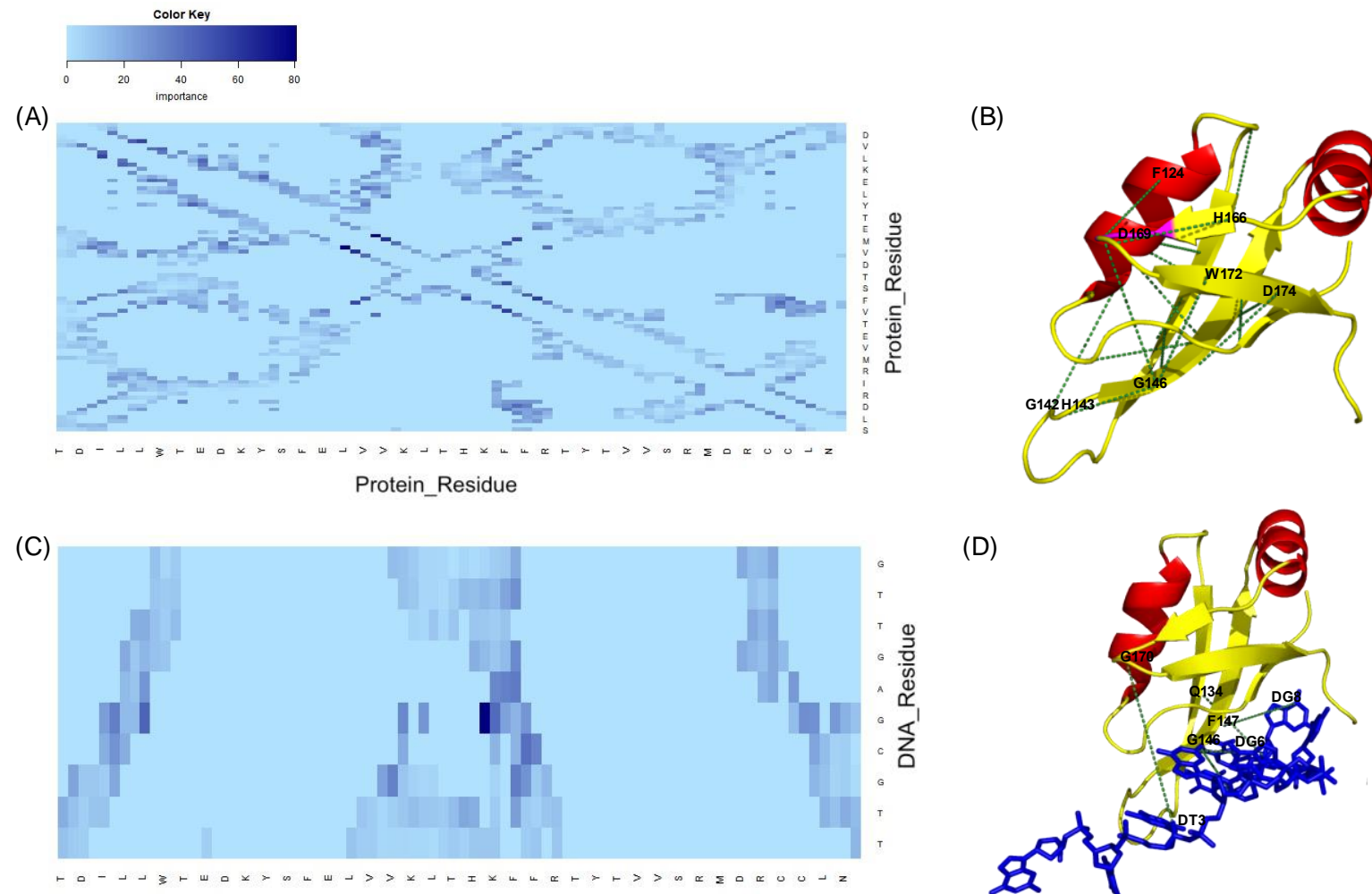

(D)

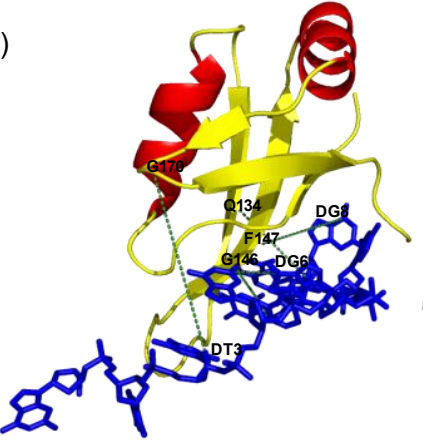

Protein_Residue

Fig.4. Global average Importance Interaction map of (A) Protein-protein interactions and (C) protein-DNA interactions. The colour intensity shows the importance of the interaction where the darker shade represents higher importance. For clear representation, every third residue in the protein sequence is labelled in the plots. Schematic representation of some of the important (B) protein-protein interactions and (D) proteinDNA interactions. The residues are represented in their single-letter amino acid code. The dashed lines indicate the interacting residue pairs.

In Fig. 4B, we have schematically shown some of the important interactions which involve the mutation site, D169 and the W172 and D174 residues in the $\beta 5$ strand of the protein where the protein is generally cleaved. We see that D169 residue has important interactions with the residues of the protein core such as G146 (shown in the diagram), W123, and also has important discriminatory interactions with the residues of loop 1 (between a1 helix and $\beta 1$ strand) such as P112. Moreover, both the mutation site residue, D169 and I168 are found to have important discriminatory interactions with T115 which is also experimentally validated to be crucial in maintaining the protein stability ${ }^{26}$. The cleavage-prone residues in the $\beta 5$ strand are found to have important interactions with the protein core residues, such as W172 - G146, F147 - D174, F149 - D174, V161 - D174. The importance of the core residues can also be seen in the interaction of the protein with the DNA where the core residues such as Q134, F147, G146 is found to have an important interaction with the DNA molecule. 


\subsubsection{Objective II- Identifying individual important residues in the RRM1 domain of TDP-43}

While unravelling the important interacting pairs, it is also necessary to identify the contribution of the individual residues of the RRM1 domain that plays a crucial role in maintaining the stability of the protein. For a given residue, its importance is calculated by averaging the importance of all the identified interaction pairs that include that particular residue. Since LASSO and XGBoost drop all the moderate to low contributing interactions, they are abysmal to depict the true per residue importance profile. Consequently, the ElasticNet and RandomForest models were employed. Interestingly, the per residue importance profile indicated that the protein core comprised of the $\beta 3$ and $\beta 4$ strand of the RRM1 motif shows the highest importance per residue (Fig. 5A). The highest importance per residue in the $\beta 3$ and $\beta 4$ strands are found for the residues Q134 and G148. Among the top 15 highest important residues (marked by dashed lines in Fig. 5A) include the I166 and I168 residues of $\beta 4$ and residues spanning the $\beta 1$ and a1 strand.

(A)

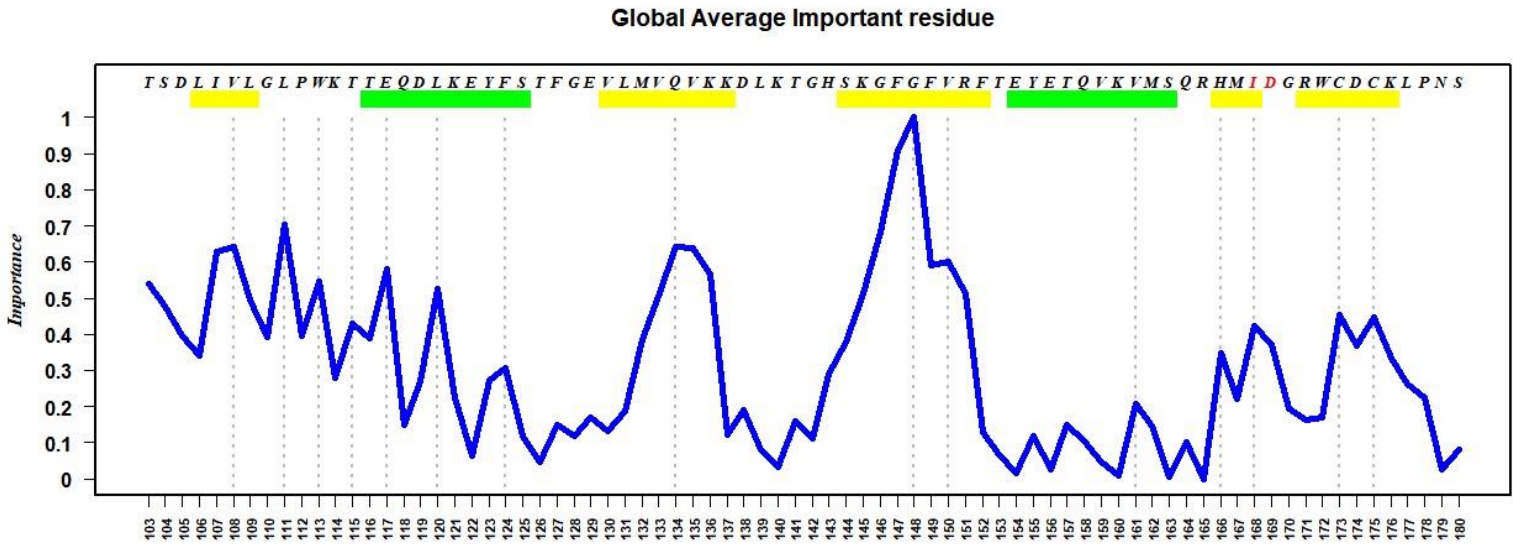

(B)
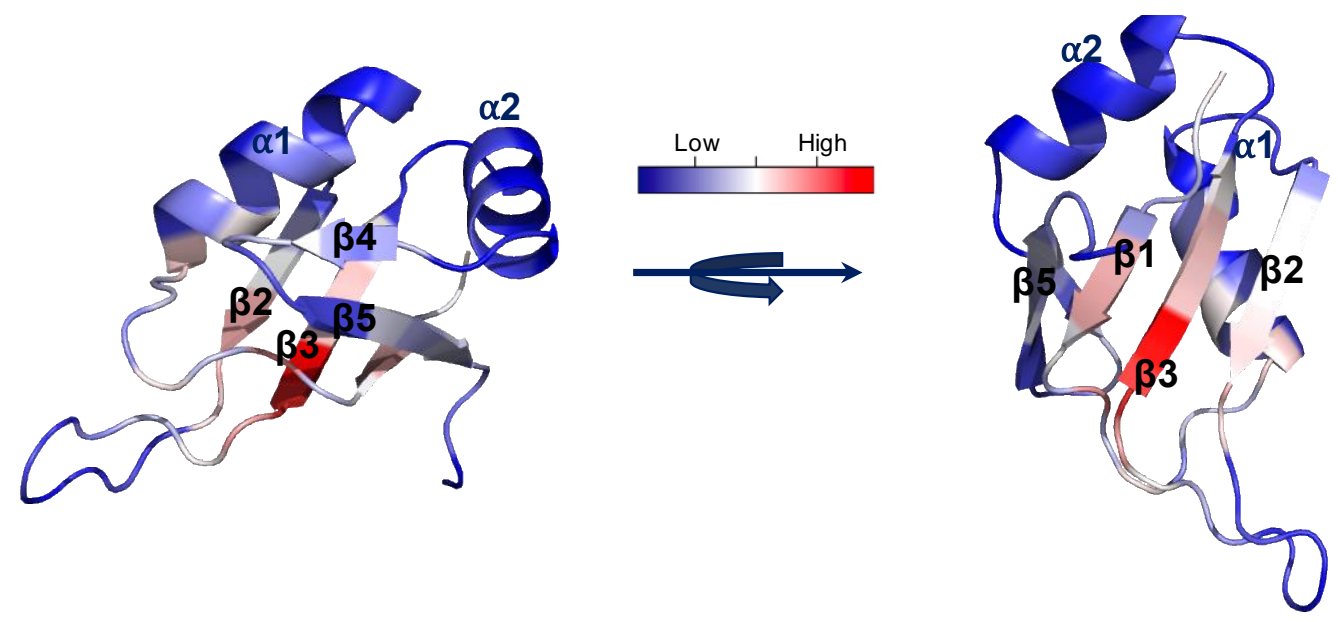

Fig. 5. (A) Global average Important residue map of all the residues in the RRM1 motif of TDP-43 protein. The green and yellow bars represent the alpha-helix and beta-strand respectively. (B) Gradient colour coded 
important residue cartoon representation of the RRM1 protein. The redder shade represents higher importance and the transition from red to blue indicates a decrease in the importance scores.

Fig. 5B shows a schematic representation of the residue importance where the redder shade represents higher importance, and the blue shade represents less importance. This also suggests that the residues in the a1 strand, especially E117 and L120 shown by lighter blue shade are as important as the mutant residues and can also influence the protein stability, which can be experimentally tested by mutagenesis studies. Although similar observation is seen for the interaction map of the individual mutant proteins [Fig. S6], the per residue importance of the protein core region is highest for the I168A-D169G double mutant as compared to the other two mutations. Hence, our analysis not only elucidates the underlying molecular basis of disease-associated mutations in the TDP-43 protein but also helps in identifying the crucial residues involved in the protein stability which can be genetically engineered to address its pathogenesis.

\section{Conclusion}

Recently, neurodegenerative disorders linking to TDP-43 malfunction have increased considerably and mutational modifications in the protein are found to expedite its toxic cytoplasmic aggregation ${ }^{8}$. In this work, we performed an extensive structural analysis on the effect of common disease-causing mutations on the RRM1 domain of TDP-43 protein combining molecular dynamics simulations with machine learning models through the MD-ML workflow. Out of the three mutations studied, D169G, I168A-D169G and I168A, we found that the I168A-D169G double mutant shows the highest packing of the protein inner core, indicating more stability and hence can lead to the enhanced level of pathogenesis which needs to be experimentally validated in the future. Moreover, using machine learning approaches, we identified the important (i) protein-protein and protein-DNA interacting pairs and (ii) individual protein residues that are crucial in maintaining the stability of the protein molecule. We showed that along with the protein residues in the mutation sites, the residues in the cleavage sites are also involved in important interactions with the protein core. Moreover, the per residue importance profile shows the crucial role of the protein inner core in maintaining the stability of the protein. In addition to that, residues in the al strand are found to be important which can be experimentally validated for their influence on the protein stability and hence its pathogenesis. This information will help biomedical researchers working in emerging strategies towards TDP-43 disaggregation and develop prospective therapeutics in the future.

\section{Declaration of competing Interests}

The authors declare they have no conflict of interests.

\section{Funding}


bioRxiv preprint doi: https://doi org/10.1101/2021.07.28.454112; this version posted July 28,2021 . The copyright holder for this preprin (which was not certified by peer review) is the author/funder, who has granted bioRxiv a license to display the preprint in perpetuity. It is made available under aCC-BY-NC-ND 4.0 International license.

This research did not receive any specific grant from funding agencies in the public, commercial, or notfor-profit sectors.

\section{References:}

1. Jo, M. et al. The role of TDP-43 propagation in neurodegenerative diseases: integrating insights from clinical and experimental studies. Experimental \& Molecular Medicine 52, 1652-1662 (2020).

2. Scotter, E.L., Chen, H.-J. \& Shaw, C.E. Erratum to: TDP-43 Proteinopathy and ALS: Insights into Disease Mechanisms and Therapeutic Targets. Neurotherapeutics 12, 515-518 (2015).

3. Nakielny, S. \& Dreyfuss, G. Nuclear export of proteins and RNAs. Curr Opin Cell Biol. 9, 420429 (1997).

4. Geuens, T., Bouhy, D. \& Timmerman, V. The hnRNP family: insights into their role in health and disease. Hum Genet 135, 851-867 (2016).

5. Inukai, Y. et al. Abnormal phosphorylation of Ser409/410 of TDP-43 in FTLD-U and ALS. FEBS Lett 582, 2899-2904 (2008).

6. Arai, T. et al. TDP-43 is a component of ubiquitin-positive tau-negative inclusions in frontotemporal lobar degeneration and amyotrophic lateral sclerosis. Biochem Biophys Res Commun 351, 602-611 (2006).

7. Hasegawa, M. et al. Phosphorylated TDP-43 in frontotemporal lobar degeneration and amyotrophic lateral sclerosis. Ann Neurol 64, 60-70 (2008).

8. Prasad, A., Bharathi, V., Sivalingam, V., Girdhar, A. \& Patel, B.K. Molecular Mechanisms of TDP43 Misfolding and Pathology in Amyotrophic Lateral Sclerosis. Frontiers in Molecular Neuroscience 12 (2019).

9. Ling, S.-C. et al. ALS-associated mutations in TDP-43 increase its stability and promote TDP-43 complexes with FUS/TLS. Proceedings of the National Academy of Sciences 107, 13318-13323 (2010).

10. Dugger, B.N. \& Dickson, D.W. Pathology of neurodegenerative diseases. Cold Spring Harb. Perspect. Biol. 9, a028035. (2017).

11. Nichols, E. et al. Global, regional, and national burden of Alzheimer's disease and other dementias, 1990-2016: a systematic analysis for the Global Burden of Disease Study 2016. The Lancet Neurology 18, 88-106 (2019).

12. Suk, T.R. \& Rousseaux, M.W.C. The role of TDP-43 mislocalization in amyotrophic lateral sclerosis. Molecular Neurodegeneration 15, 45 (2020).

13. Zhang, Y.J. et al. Aberrant cleavage of TDP-43 enhances aggregation and cellular toxicity. Proc Natl Acad Sci U S A 106, 7607-7612 (2009).

14. McAlary, L., Plotkin, S.S., Yerbury, J.J. \& Cashman, N.R. Prion-Like Propagation of Protein Misfolding and Aggregation in Amyotrophic Lateral Sclerosis. Front Mol Neurosci 12, 262 (2019).

15. Nonaka, T., Watanabe, S.T., Iwatsubo, T. \& Hasegawa, M. Seeded aggregation and toxicity of \{alpha\}-synuclein and tau: cellular models of neurodegenerative diseases. $J$ Biol Chem 285, 3488534898 (2010).

16. Freibaum, B.D., Chitta, R.K., High, A.A. \& Taylor, J.P. Global Analysis of TDP-43 Interacting Proteins Reveals Strong Association with RNA Splicing and Translation Machinery. Journal of Proteome Research 9, 1104-1120 (2010).

17. Polymenidou, M. et al. Misregulated RNA processing in amyotrophic lateral sclerosis. Brain Research 1462, 3-15 (2012).

18. Johnson, B.S. et al. TDP-43 Is Intrinsically Aggregation-prone, and Amyotrophic Lateral Sclerosislinked Mutations Accelerate Aggregation and Increase Toxicity *. Journal of Biological Chemistry 284, 20329-20339 (2009).

19. Mutihac, R. et al. TARDBP pathogenic mutations increase cytoplasmic translocation of TDP-43 and cause reduction of endoplasmic reticulum $\mathrm{Ca} 2+$ signaling in motor neurons. Neurobiology of Disease 75, 64-77 (2015).

20. Neumann, M. et al. Ubiquitinated TDP-43 in frontotemporal lobar degeneration and amyotrophic lateral sclerosis. Science 314, 130-133 (2006). 
21. Yamashita, T. et al. A role for calpain-dependent cleavage of TDP-43 in amyotrophic lateral sclerosis pathology. Nature Communications 3, 1307 (2012).

22. De Marco, G. et al. Reduced cellular Ca2+ availability enhances TDP-43 cleavage by apoptotic caspases. Biochimica et Biophysica Acta (BBA) - Molecular Cell Research 1843, 725-734 (2014).

23. Li, Q., Yokoshi, M., Okada, H. \& Kawahara, Y. The cleavage pattern of TDP-43 determines its rate of clearance and cytotoxicity. Nature Communications 6, 6183 (2015).

24. Xiao, S. et al. Low molecular weight species of TDP-43 generated by abnormal splicing form inclusions in amyotrophic lateral sclerosis and result in motor neuron death. Acta Neuropathologica 130, 49-61 (2015).

25. Suzuki, H., Lee, K. \& Matsuoka, M. TDP-43-induced Death Is Associated with Altered Regulation of BIM and Bcl-xL and Attenuated by Caspase-mediated TDP-43 Cleavage Journal of Biological Chemistry 286, 13171-13183 (2011).

26. Chiang, C.-H. et al. Structural analysis of disease-related TDP-43 D169G mutation: linking enhanced stability and caspase cleavage efficiency to protein accumulation. Scientific Reports $\mathbf{6}$, 21581 (2016).

27. Che, M.X., Jiang, L.L., Li, H.Y., Jiang, Y.J. \& Hu, H.Y. TDP-35 sequesters TDP-43 into cytoplasmic inclusions through binding with RNA. FEBS Lett 589, 1920-1928 (2015).

28. Cicardi, M.E. et al. Tdp-25 Routing to Autophagy and Proteasome Ameliorates its Aggregation in Amyotrophic Lateral Sclerosis Target Cells. Scientific reports 8, 12390 (2018).

29. Kitamura, A. et al. Interaction of RNA with a C-terminal fragment of the amyotrophic lateral sclerosis-associated TDP43 reduces cytotoxicity. Scientific reports 6, 19230 (2016).

30. Huang, Y.-C. et al. Inhibition of TDP-43 Aggregation by Nucleic Acid Binding. PLOS ONE 8, e64002 (2013).

31. Noé, F. \& Clementi, C. Collective variables for the study of long-time kinetics from molecular trajectories: theory and methods. Current Opinion in Structural Biology 43, 141-147 (2017).

32. Lundervold, A.S. \& Lundervold, A. An overview of deep learning in medical imaging focusing on MRI. Zeitschrift für Medizinische Physik 29, 102-127 (2019).

33. Senior, A.W. et al. Improved protein structure prediction using potentials from deep learning. Nature 577, 706-710 (2020).

34. Sharma, A. \& Dey, P. A machine learning approach to unmask novel gene signatures and prediction of Alzheimer's disease within different brain regions. Genomics 113, 1778-1789 (2021).

35. Grear, T., Avery, C., Patterson, J. \& Jacobs, D.J. Molecular function recognition by supervised projection pursuit machine learning. Scientific Reports 11, 4247 (2021).

36. Fleetwood, O., Kasimova, M.A., Westerlund, A.M. \& Delemotte, L. Molecular Insights from Conformational Ensembles via Machine Learning. Biophysical Journal 118, 765-780 (2020).

37. Duan, Y. et al. A point-charge force field for molecular mechanics simulations of proteins based on condensed-phase quantum mechanical calculations. J Comput Chem 24, 1999-2012 (2003).

38. Parrinello, M. \& Rahman, A. Crystal Structure and Pair Potentials: A Molecular-Dynamics Study. Physical Review Letters 45, 1196-1199 (1980).

39. Kasahara, K., Fukuda, I. \& Nakamura, H. A Novel Approach of Dynamic Cross Correlation Analysis on Molecular Dynamics Simulations and Its Application to Ets1 Dimer-DNA Complex. PLOS ONE 9, e112419 (2014).

40. Hua, J., Liu, H., Zhang, B. \& Jin, S. LAK: lasso and K-means based single-cell RNA-Seq data clustering analysis. IEEE Access 8, 129679-129688 (2020).

41. Zou, H. \& Hastie, T. Regularization and Variable Selection via the Elastic Net. Journal of the Royal Statistical Society. Series B (Statistical Methodology) 67, 301-320 (2005).

42. Ghosh Roy, G., Geard, N., Verspoor, K. \& He, S. PoLoBag: Polynomial Lasso Bagging for signed gene regulatory network inference from expression data. Bioinformatics 36, 5187-5193 (2020).

43. Kuhn, M. caret: Classification and Regression Training. (2020).

44. Breiman, L. Random Forests. Machine Learning 45, 5-32 (2001).

45. Breiman, L. Bagging predictors. Machine Learning 24, 123-140 (1996).

46. Liaw, A. \& Wiener, M. Classification and regression by randomForest. Rnews 2, 18-22 (2002).

47. Jerome, H.F. Greedy function approximation: A gradient boosting machine. The Annals of Statistics 29, 1189-1232 (2001). 
bioRxiv preprint doi: https://doi org/10.1101/2021.07.28.454112 this version posted July 28 2021. The copyriaht holder for this preprin (which was not certified by peer review) is the author/funder, who has granted bioRxiv a license to display the preprint in perpetuity. It is made available under aCC-BY-NC-ND 4.0 International license.

48. Chen, T. \& Guestrin, C. in Proceedings of the 22nd ACM SIGKDD International Conference on Knowledge Discovery and Data Mining 785-794 (Association for Computing Machinery, San Francisco, California, USA; 2016).

49. $\mathrm{Bi}$, Y. et al. An Interpretable Prediction Model for Identifying $\mathrm{N}<\sup >7</$ sup $>$-Methylguanosine Sites Based on XGBoost and SHAP. Molecular Therapy - Nucleic Acids 22, 362-372 (2020).

50. Chen, X., Wang, Z.-X. \& Pan, X.-M. HIV-1 tropism prediction by the XGboost and HMM methods. Scientific Reports 9, 9997 (2019).

51. Li, W., Yin, Y., Quan, X. \& Zhang, H. Gene Expression Value Prediction Based on XGBoost Algorithm. Frontiers in Genetics 10 (2019).

52. Tianqi, C. \& Tong, H. xgboost: eXtreme Gradient Boosting. R Package Version: 1.4.1.1 (2021).

53. Amadei, A., Linssen, A.B. \& Berendsen, H.J. Essential dynamics of proteins. Proteins 17, 412-425 (1993).

54. Amadei, A., Linssen, A.B.M., de Groot, B.L., van Aalten, D.M.F. \& Berendsen, H.J.C. An Efficient Method for Sampling the Essential Subspace of Proteins. Journal of Biomolecular Structure and Dynamics 13, 615-625 (1996).

55. Tai, K., Shen, T., Börjesson, U., Philippopoulos, M. \& McCammon, J.A. Analysis of a 10-ns Molecular Dynamics Simulation of Mouse Acetylcholinesterase. Biophysical Journal 81, 715-724 (2001).

56. The PyMOL Molecular Graphics System. Schrödinger, LLC Version 1.2r3pre.

57. Austin, J.A. et al. Disease causing mutants of TDP-43 nucleic acid binding domains are resistant to aggregation and have increased stability and half-life. Proceedings of the National Academy of Sciences 111, 4309-4314 (2014).

58. Ayala, Y.M. et al. Structural determinants of the cellular localization and shuttling of TDP-43. Journal of cell science 121, 3778-3785 (2008). 


\section{Supplementary for}

\section{Unravelling the effects of disease associated mutations in TDP-43 protein via molecular dynamics simulation and machine learning.}

Abhibhav Sharma, Pinki Dey

\section{The Random Forest (RF)}

Let the feature dataset used for training is bootstrapped $B=\left\{\left(F_{i}, S_{i}\right)_{i=1}^{N} \mid F_{i} \in \mathrm{R}^{M}, S \in\{1,2, \ldots, c\}\right\}$, where $F_{i}$ are the feature set (variables) and $S$ represents its respective label (the mutation state). Let $N$ and $M$ denotes the cardinality of samples used for training and the features respectively. For $F$ be a given input instance and the prediction of the $K^{\text {th }}$ tree $T_{k}$ is represented as $\widehat{S^{k}}$. The prediction obtained by the RF as an ensemble of $K$ is:

$$
\hat{S}=\max \text { vote }\left\{\widehat{S^{k}}\right\}_{1}^{K}
$$

\section{Pseudo-Code}

The input be the bootstrapped training dataset $B$ $\mid$ mtry $\mid=$ subspace size, $|K|=$ number of tress

- For $k \rightarrow 1$ to $K$ do:
a. $\mathrm{B}_{k}$ samples are selected from the input to produce bootstrapped $B$
b. mtry features are chosen at random.
c. For $m \rightarrow 1$ to $\| m$ try $\|$ do
d. The amount of decrease in the node impurity (Gini Impurity) is calculate
e. Most contributing variable in the impurity decline is chosen and the node is then split into two daughter/child nodes

- The ensemble of the $K$ trees produce a RF

While bootstrapping the feature dataset, due to the sampling with replacement not all the samples were used to prune the tree. These samples are called the in-bag samples. The left-out instances are coined as out-ofbag (OOB) sample. The OOB samples are exploited to calculate the error in prediction for each generated random forest also called as the OOB error rate.

The OOB value is given as:

$\hat{S}^{O O B}=\left(\frac{1}{\left\|\theta_{i^{\prime}}\right\|}\right) \sum_{k \epsilon \theta_{i}} \hat{S}^{k}$, where $\theta_{i^{\prime}}=\frac{\mathrm{B}}{\theta_{i}}, \mathrm{i}^{\prime}$ and $i$ denotes the out-of-bag and in-bag sampled instances, $\left\|\theta_{i}\right\|$ is the number of $\mathrm{OOB}$ instances. The $\mathrm{OOB}$ prediction error is:

$$
\widehat{\operatorname{Err}}^{O O B}=\frac{1}{N_{O O B}} \sum_{i=1}^{N_{O O B}} \Psi\left(\mathrm{S}, \hat{S}^{O O B}\right)
$$

Here $\Psi($.$) is the error function and N_{O O B}$ is OOB sample's size. 


\section{Gradient Boosting Algorithm}

Boosting algorithms are gravitating a lot of attention in recent years. Of this, gradient boosting algorithm (GBM) are classification and regression models, that generates a prediction model through an ensemble of weak decision tress. Although a tree-based model, GBM outperforms the random forest models in terms of accuracy and speed $[1,2,3]$. The GBM model is built in a stage-wise fashion while introducing an arbitrary differentiable loss function [4,5]. Friedman introduced GBM models for regression and from there on the regularization and generalization of GBM emerged [6,7].

Here we state the pseudocode of a GBM machine. A preeminent view of GBM can be found in Friedman et al. [5].

Let the Data set be $X$ as $\left\{\left(x_{i}, y_{i}\right)\right\}_{i=1}^{n}$. Let the differentiable loss function be defined as $L(y, F(x))$ and the number of iterations be $M$

The algorithm then follows:

- The model is initialized as constant value. The constant here is basically the mean target value.

$$
F_{0}(x)=\arg \min _{\gamma} \sum_{i=1}^{n} L\left(y_{i}, \gamma\right)
$$

- $\quad$ For $m=1$ to $M$ :

- The pseudo-residuals are calculated as:

$$
r_{i m}=\left[\frac{\partial L\left(y_{i}, F\left(x_{i}\right)\right)}{\partial F\left(x_{i}\right)}\right]_{F(x)=F_{m-1}(x)} \quad \text { For } i=1 \ldots n
$$

- The weak learner is fitted closed under the scaling of the calculated pseudo-residuals $h_{m}(x)$. This mean that the training is carried out over the new derived data set $\left\{\left(x_{i}, r_{i m}\right)\right\}_{i=1}^{n}$.

- By solving the optimization problem, the $\gamma_{m}$ is calculates:

$$
\gamma_{m}=\arg _{\gamma} \min \sum_{i=1}^{n} L\left(y_{i}, F_{m-1}\left(x_{i}\right)+\gamma h_{m}\left(x_{i}\right)\right)
$$

- The model is the updated

$$
F_{m}(x)=F_{m-1}(x)+\gamma_{m} h_{m}(x)
$$

- $\quad$ Output $F_{M}(x)$ 
bioRxiv preprint doi: https://doi.org/10.1101/2021.07.28.454112; this version posted July $28,2021$. The copyright holder for this preprint (which was not certified by peer review) is the author/funder, who has granted bioRxiv a license to display the preprint in perpetuity. It is made available under aCC-BY-NC-ND 4.0 International license.

\section{Table}

\begin{tabular}{|c|c|c|c|c|c|}
\hline Model & \multicolumn{1}{|c}{ Mutation Type } & \multicolumn{1}{c}{ Accuracy } & \multicolumn{2}{c|}{ Sensitivity } & \multicolumn{2}{c|}{ Specificity } & Precision \\
\hline \multirow{3}{*}{ Random Forest } & D168G & 0.999 & 0.998 & 1.000 & 1.000 \\
\cline { 2 - 6 } & D168G_I169A & 1.000 & 1.000 & 1.000 & 1.000 \\
\cline { 2 - 6 } & I169A & 0.996 & 0.992 & 1.000 & 1.000 \\
\hline \multirow{3}{*}{ Elastic Net } & D168G & 1.000 & 1.000 & 1.000 & 1.000 \\
\cline { 2 - 6 } & D168G_I169A & 1.000 & 1.000 & 1.000 & 1.000 \\
\cline { 2 - 6 } & I169A & 0.995 & 0.990 & 1.000 & 1.000 \\
\hline \multirow{3}{*}{ LASSO } & D168G & 1.000 & 1.000 & 1.000 & 1.000 \\
\cline { 2 - 6 } & D168G_I169A & 1.000 & 1.000 & 1.000 & 1.000 \\
\cline { 2 - 6 } & I169A & 0.999 & 0.998 & 1.000 & 1.000 \\
\hline \multirow{3}{*}{ XGBoost } & D168G & 1.000 & 1.000 & 1.000 & 1.000 \\
\cline { 2 - 6 } & D168G_I169A & 1.000 & 1.000 & 1.000 & 1.000 \\
\cline { 2 - 6 } & I169A & 0.997 & 0.995 & 1.000 & 1.000 \\
\hline
\end{tabular}

Table S1. The classification performance metric of all the models for each type of mutation type. Accuracy = $\mathrm{TP}+\mathrm{TN} /(\mathrm{TP}+\mathrm{TN}+\mathrm{FP}+\mathrm{FN})$, Sensitivity $=\mathrm{TP} /(\mathrm{TP}+\mathrm{FN})$, Specificity $=\mathrm{TN} /(\mathrm{TN}+\mathrm{FP})$ and Precision $=\mathrm{TP} /(\mathrm{TP}+\mathrm{FP})$ where TP, TN, FP and FN are True Positive, True Negative, False positive and False Negative classifications respectively.

\section{Hyperparameter Table}

\begin{tabular}{|c|l|l|}
\hline Model & \multicolumn{1}{|c|}{ Critical Parameters } & \multicolumn{1}{c|}{ Note } \\
\hline LASSO & $\begin{array}{l}\text { method }=\text { "glmnet", } \\
\text { lambda= seq(0.0001,1, length =5) }\end{array}$ & $\begin{array}{l}\text { The alpha is not declared, setting 0 } \\
\text { by default thus performing LASso }\end{array}$ \\
\hline RandomForest & $\begin{array}{l}\text { ntree=300, } \\
\text { mtry=8 }\end{array}$ & $\begin{array}{l}\text { ntree employed here is the default value } \\
\text { which was tested against 250, 300 and 400. }\end{array}$ \\
\hline Elastic Net & $\begin{array}{l}\text { method }=\text { "glmnet", } \\
\text { alpha= seq(0,1,length=10), } \\
\text { lambda= seq(0.0001,1, length =5) }\end{array}$ & $\begin{array}{l}\text { Both aplha and beta were } \\
\text { searched within the given possible set }\end{array}$ \\
\hline XGBoost & $\begin{array}{l}\text { Objective }=\text { Multi:softprob, } \\
\text { eval_metric =mlogloss, } \\
\text { num_class }=4, \\
\text { nrounds }=1000, \\
\text { eta }=0.01, \\
\text { max.depth }=3, \\
\text { gamma=0, } \\
\text { subsample=1 }\end{array}$ & The other parameters were kept in default \\
\hline
\end{tabular}

Table S2. The hyperparameters employed for the machine learning models. 
bioRxiv preprint doi: https://doi.org/10.1101/2021.07.28.454112; this version posted July 28,2021 . The copyright holder for this preprin (which was not certified by peer review) is the author/funder, who has granted bioRxiv a license to display the preprint in perpetuity. It is made available under aCC-BY-NC-ND 4.0 International license.
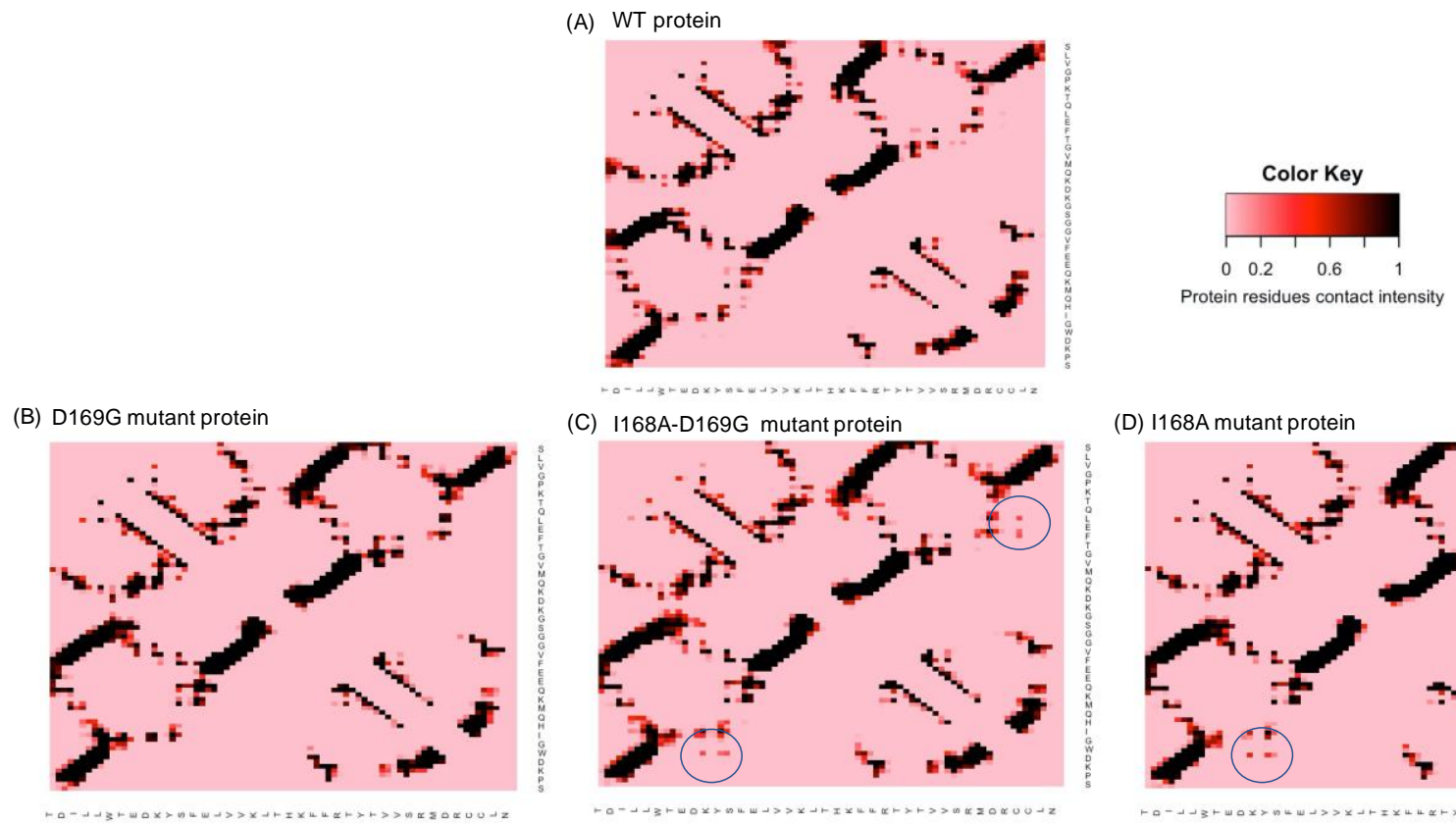

(C) I168A-D169G mutant protein
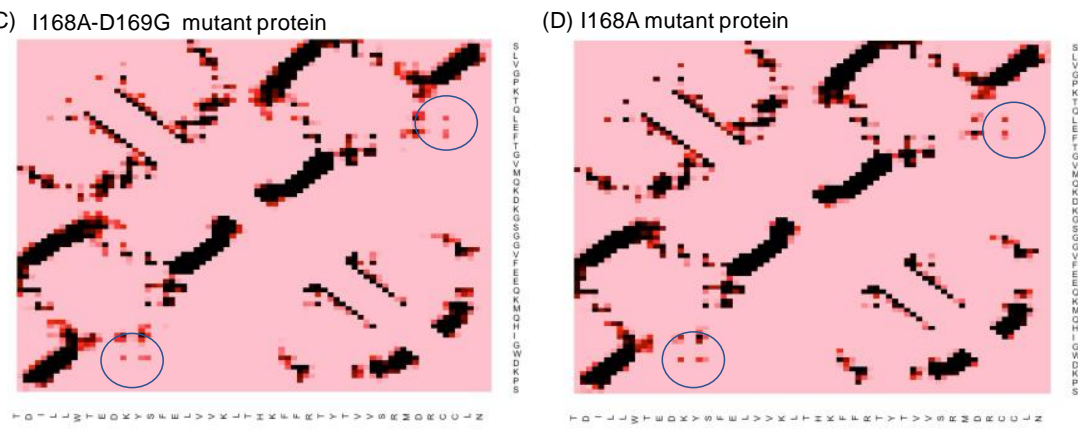

Fig. S1. Contact map for (A) Wild-type (B) D169G (C) I168A-D169G (D) I168A proteins calculated throughout the simulation time. The darker the spot in the contact map, the longer the contact was formed between the pair of residues.

(A) WT protein

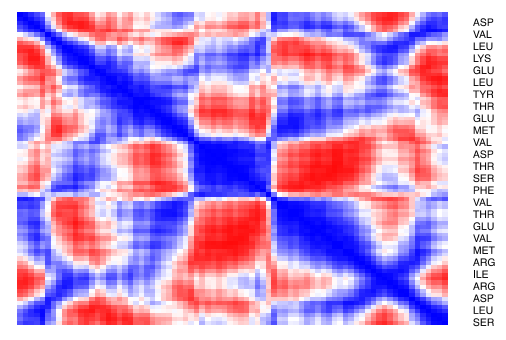

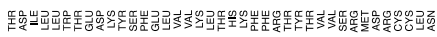

(C) I168A-D169G mutant protein

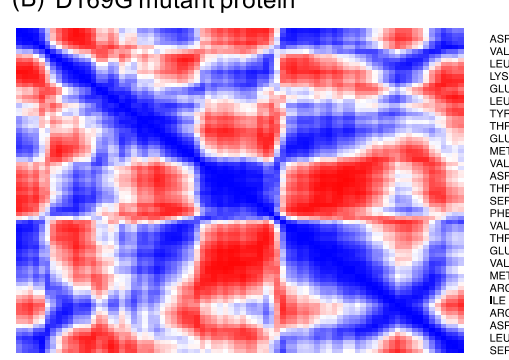

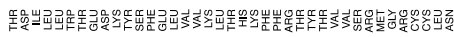

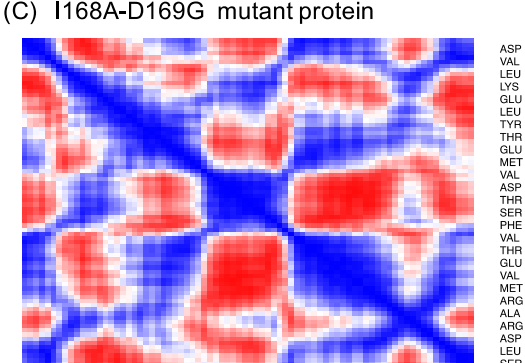

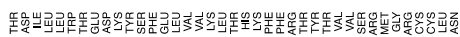

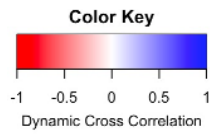

(D) I168A mutant protein

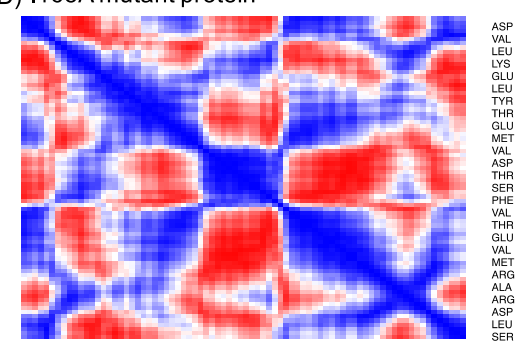

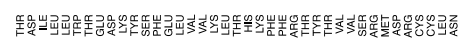

Fig. S2. Dynamic cross-correlation map of (A) Wild-type (B) D169G (C) I168A-D169G (D) I168A proteins throughout the simulation time. The negative value represents anti-correlated motion whereas the positive value represents correlated motion. 
bioRxiv preprint doi: https://doi.org/10.1101/2021.07.28.454112; this version posted July 28, 2021. The copyright holder for this preprin (which was not certified by peer review) is the author/funder, who has granted bioRxiv a license to display the preprint in perpetuity. It is made available under aCC-BY-NC-ND 4.0 International license.

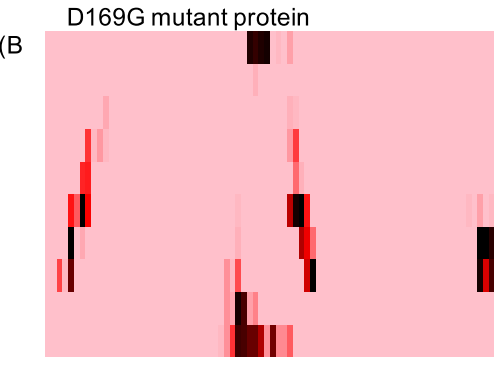

Protein_Residue

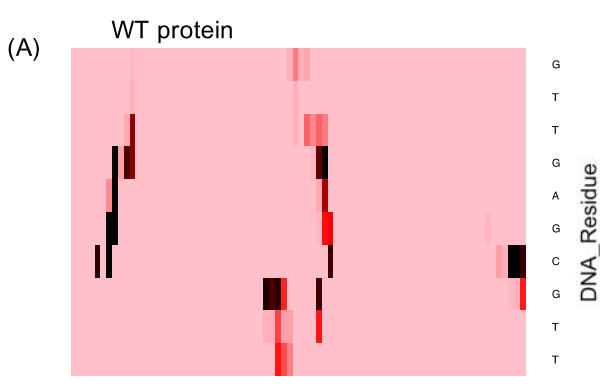

Protein_Residue

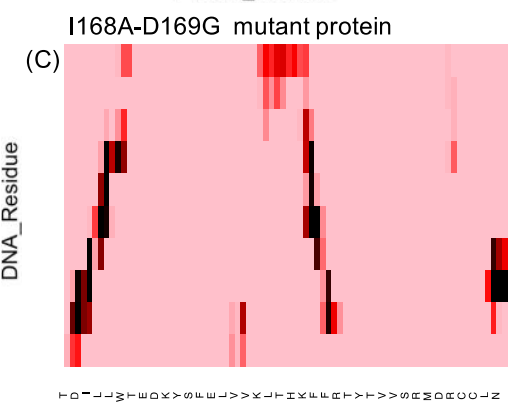

Protein_Residue
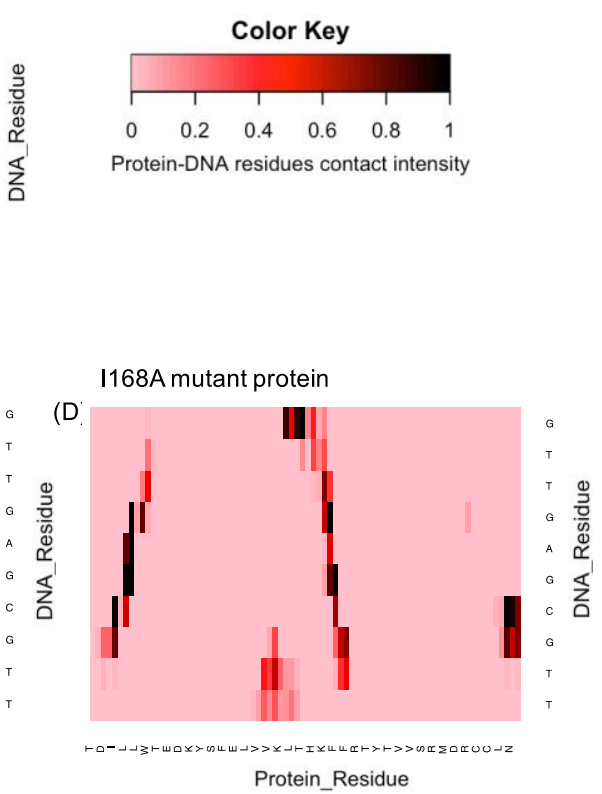

Fig. S3. Protein-DNA contact map for (A) Wild-type (B) D169G (C) I168A-D169G (D) I168A proteins throughout the simulation time. The darker the spot in the contact map, the longer the contact was formed between the pair of residues.

\section{ROC AUC}

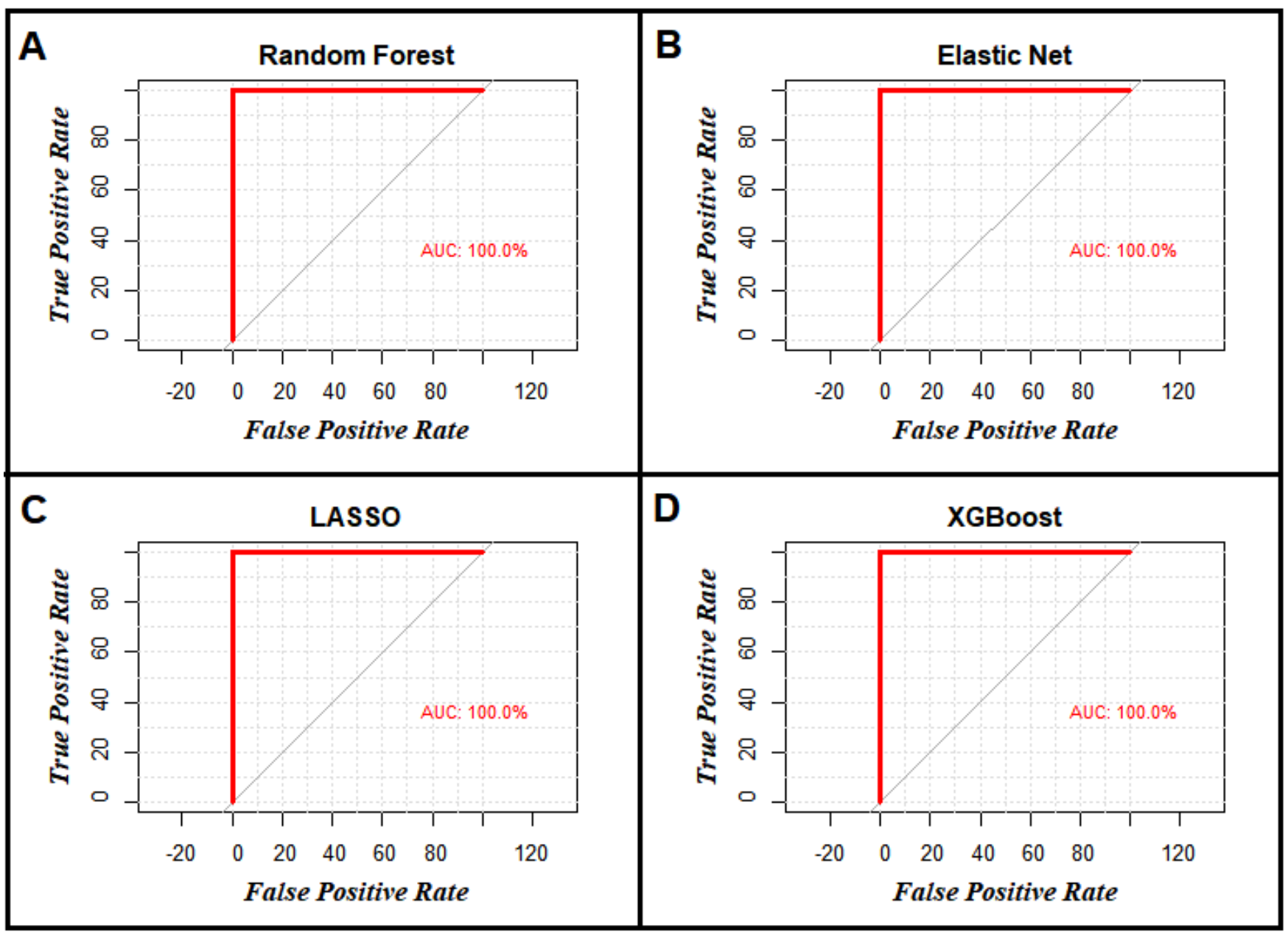


bioRxiv preprint doi: https://doi.org/10.1101/2021.07 28.454112; this version posted July 28, 2021. The copyright holder for this preprint (which was not certified by peer review) is the author/funder, who has granted bioRxiv a license to display the preprint in perpetuity. It is made available under aCC-BY-NC-ND 4.0 International license.

Fig. S4. The ROC curve for each type of model (A) RandomForest, (B) Elastic Net, (C) LASSO, (D) XGBoost; plotted for the five-fold test dataset. The AUC (Area Under Curve) is observed to be 100\% for all models when trained for all labelled mutated states.

(A)

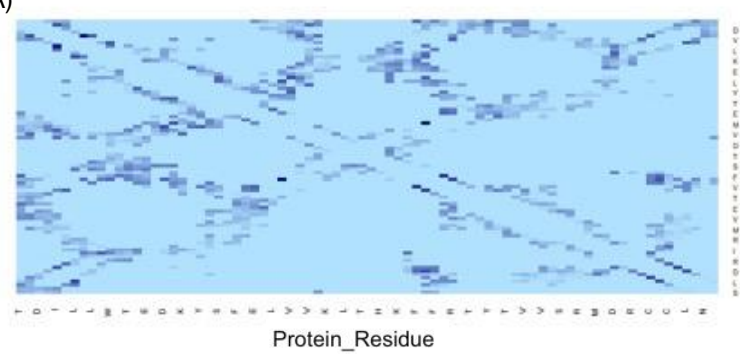

(C)

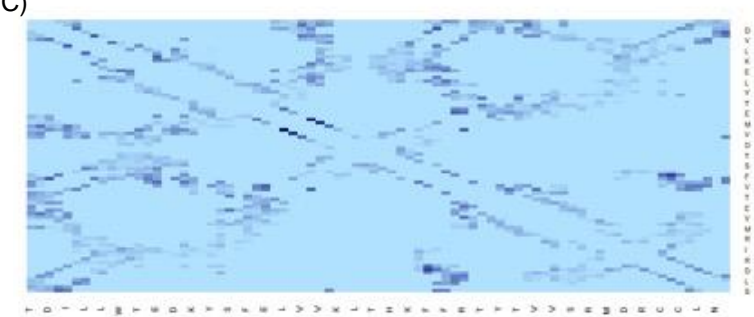

$(\mathrm{E})$

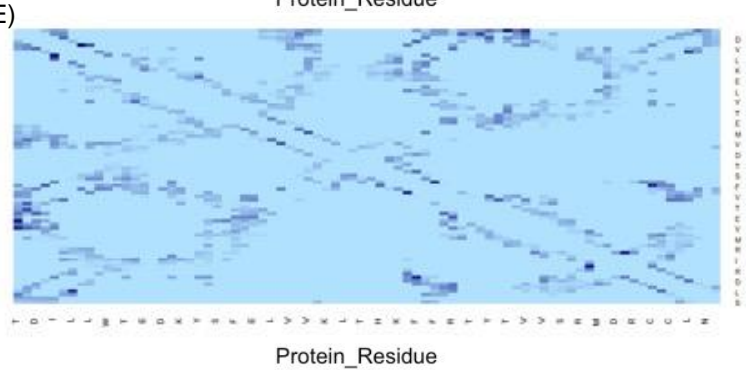

(B)

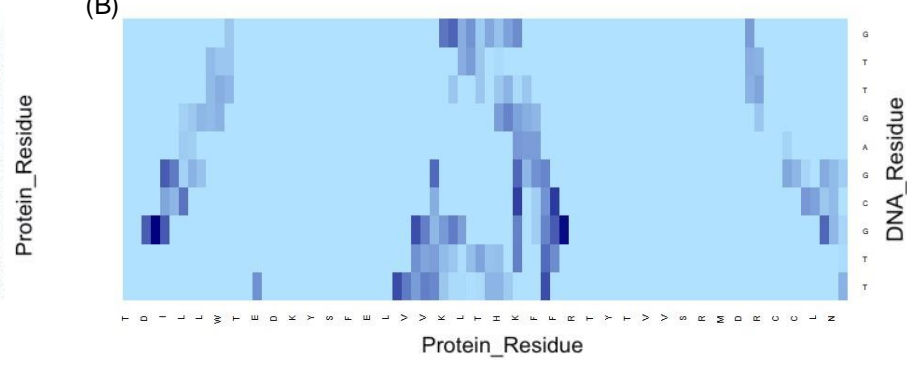

(D)

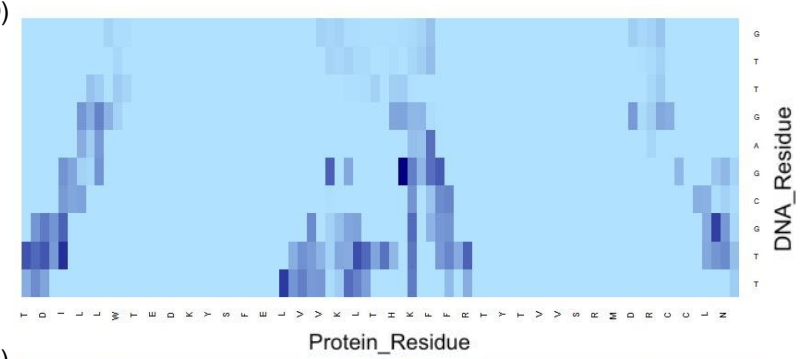

(F)

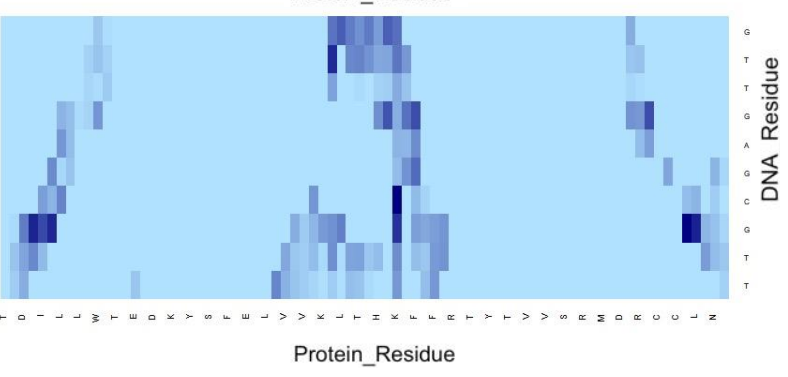

Fig. S5. Importance Interaction map of Protein-protein interactions (A,C,E) and protein-DNA interactions(B, D, F) for (A,B) D169G (C,D) I168A-D169G (E,F) I168A mutant proteins. The colour intensity shows the importance of the interaction where the darker shade represents higher importance. For clear representation, ever third residue in the protein sequence are labelled in the plots. 
(A)

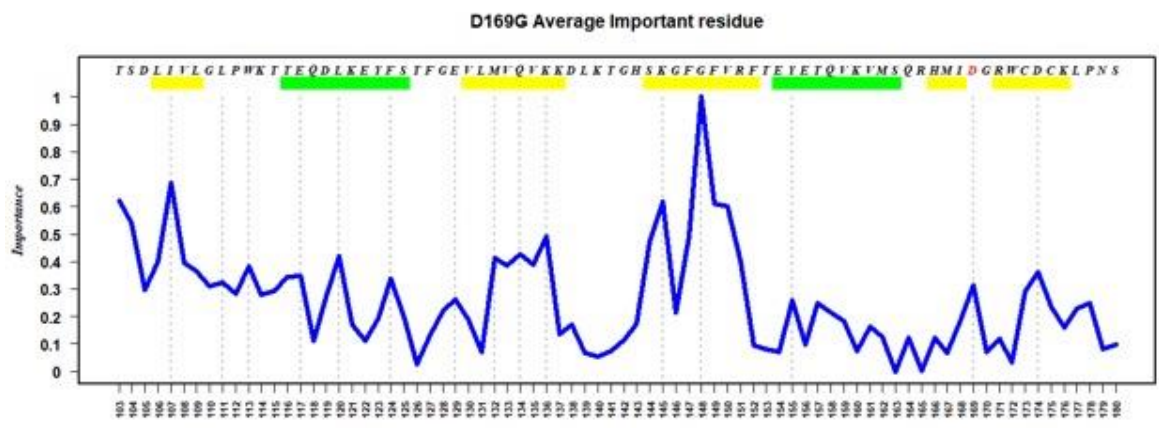

(B)

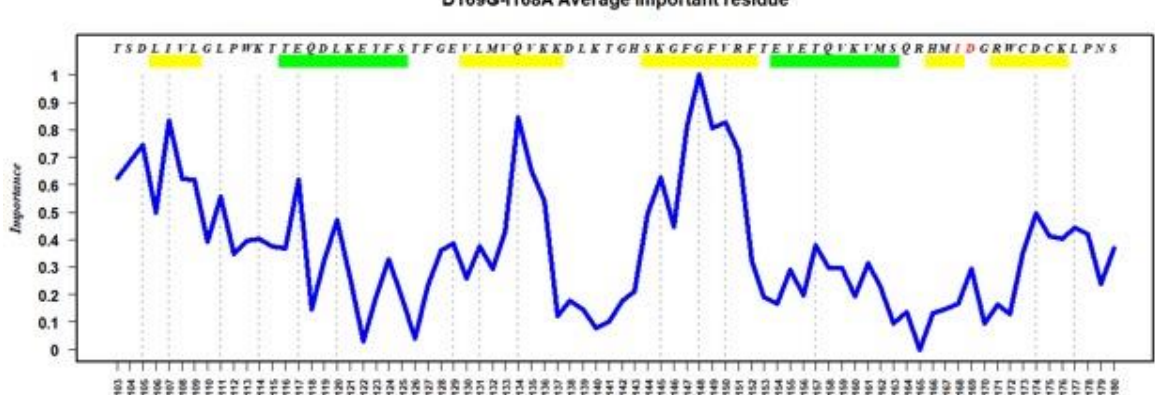

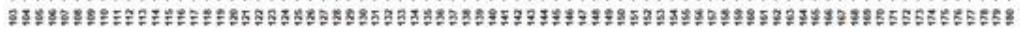

(C)

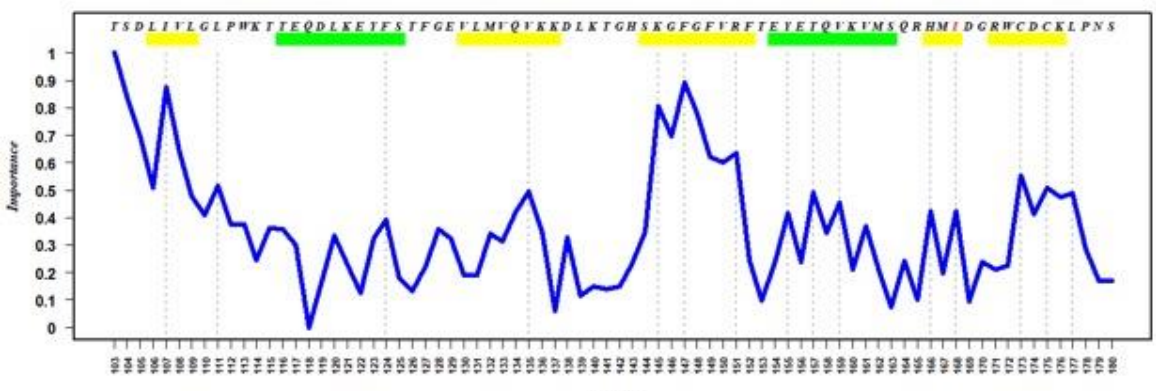

Fig. S6. Important residue map of all the residues in the RRM1 motif for (A) D169G (B) I168AD169G (C) I168A proteins. The green and yellow bars represent the alpha helix and beta strand respectively.

\section{References}

1. Piryonesi, S. Madeh; El-Diraby, Tamer E. "Data Analytics in Asset Management: Cost-Effective Prediction of the Pavement Condition Index". Journal of Infrastructure Systems.2020, 26 (1): 04019036.

2. Piryonesi, S. Madeh; El-Diraby, Tamer E. "Using Machine Learning to Examine Impact of Type of Performance Indicator on Flexible Pavement Deterioration Modeling". Journal of Infrastructure Systems.2021 27 (2): 04021005.

3. Hastie, T.; Tibshirani, R.; Friedman, J. H. "10. Boosting and Additive Trees". The Elements of Statistical Learning (2nd ed.).New York: Springer. pp. 2009, 337-384.

4. Friedman, J. H. "Greedy Function Approximation: A Gradient Boosting Machine".1999.

5. Friedman, J. H. "Stochastic Gradient Boosting". 1999.

6. Mason, L.; Baxter, J.; Bartlett, P. L.; Frean, Marcus. "Boosting Algorithms as Gradient Descent" (PDF). In S.A. Solla and T.K. Leen and K. Müller (ed.). Advances in Neural Information Processing Systems 12. MIT Press. 1999, 512-518.

7. Mason, L.; Baxter, J.; Bartlett, P. L.; Frean, Marcus. "Boosting Algorithms as Gradient Descent in Function Space".1999. 